\title{
PERIODIC ORBITS FROM SECOND ORDER PERTURBATION VIA RATIONAL TRIGONOMETRIC INTEGRALS
}

\author{
R. PROHENS AND J. TORREGROSA
}

\begin{abstract}
The second order Poincaré-Pontryagin-Melnikov perturbation theory is used in this paper to study the number of bifurcated periodic orbits from certain centers. This approach also allows us to give the shape and the period up to first order. We address these problems for some classes of Abel differential equations and quadratic isochronous vector fields in the plane. We prove that two is the maximum number of hyperbolic periodic orbits bifurcating from the isochronous quadratic centers with a birational linearization under quadratic perturbations of second order. In particular the configurations $(2,0)$ and $(1,1)$ are realizable when two centers are perturbed simultaneously. The required computations show that all the considered families share the same iterated rational trigonometric integrals.
\end{abstract}

\section{INTRODUCTION}

In this paper we study the number, the shape and the period of closed trajectories bifurcating from the period annuli for some families of differential equations. We focus our attention on the second order analysis of the perturbed equation

$$
\frac{d r}{d \theta}=a_{0}(r, \theta)+\varepsilon a_{1}(r, \theta)+\varepsilon^{2} a_{2}(r, \theta)+O\left(\varepsilon^{3}\right) .
$$

Here $a_{i}$ is an analytic function, $2 \pi$-periodic in $\theta \in[-\pi, \pi]$, for $i=0,1,2$. We denote by $r(\theta ; \rho, \varepsilon)$ the solution of (1) satisfying $r(-\pi ; \rho, 0)=\rho$. We will assume throughout this paper that $\varepsilon$ is small enough. The power series in $\varepsilon$ of this solution is written as

$$
r(\theta ; \rho, \varepsilon)=r_{0}(\theta ; \rho)+\varepsilon r_{1}(\theta ; \rho)+\varepsilon^{2} r_{2}(\theta ; \rho)+O\left(\varepsilon^{3}\right) .
$$

We refer to this expansion as the shape of the orbit. Additionally we assume that, when $\varepsilon=0$, equation (1) has a period annulus; that is, an open continuum neighborhood of periodic solutions. In particular, there exists an open interval where the function $r_{0}(\theta ; \rho)$ is $2 \pi$-periodic in $\theta \in[-\pi, \pi]$, for every $\rho$ in this interval.

In the concrete case $a_{0}(r, \theta)=a(\theta) r^{2}$ we have $r_{0}(\theta ; \rho)=\rho /(1-\rho A(\theta))$ where $A(\theta)=\int_{-\pi}^{\theta} a(\psi) d \psi$. Note that $A$ is also an analytic $2 \pi$-periodic function. Furthermore, in a first order analysis of the first return map associated to the period

2010 Mathematics Subject Classification. Primary 34C07, Secondary: 37G15, 34C23, 34C25.

Key words and phrases. Polynomial differential equation; Abel equation; Bifurcation of periodic orbits; Number, shape and period of periodic solutions; First and second order perturbation; Isochronous quadratic centers; Simultaneous bifurcation. 
annulus, the Poincaré-Pontryagin-Melnikov function takes the form

$$
F_{1}(\rho)=\int_{-\pi}^{\pi} a_{1}\left(\frac{\rho}{1-\rho A(\theta)}, \theta\right)(1-\rho A(\theta))^{2} d \theta=\sum_{i=0}^{\infty} \int_{-\pi}^{\pi} \frac{a_{1, i}(\theta) \rho^{i}}{(1-\rho A(\theta))^{i-2}} d \theta,
$$

when $a_{1}(r, \theta)=\sum_{i=0}^{\infty} a_{1, i}(\theta) r^{i}$. It is well known that each simple zero of $F_{1}$ give rise to a $2 \pi$-periodic solution bifurcating from $r_{0}(\theta ; \rho)$, see [31].

Observe that $F_{1}$ is defined through an Abelian integral and, in general, the computation of this type of integrals is quite difficult because it involves transcendental functions. As it can be seen in $(3)$, to obtain $F_{1}$ we integrate rational trigonometric functions and hence some non-rational terms appear in the calculation but, since we integrate over the usual circles and by using the formulas of Appendix A, we have their expressions.

Also it is known that if this integral is vanishing identically then, in the second order analysis, the Poincaré-Pontryagin-Melnikov function is no longer an Abelian integral, see [14, 16, 20,21], and moreover its study involves the computation of the also called iterated integrals in the sense of Chen-Gavrilov, see [14]. Concerning the infinitesimal Hilbert's 16th problem this case is a more interesting one since the Poincaré-Pontryagin-Melnikov function can have more zeros, i.e. we can produce (in general) more limit cycles than at the ones at first order, see [15, 18, 19]. More details about higher order Poincaré-Pontryagin-Melnikov theory can be found in [7].

In this paper we focus on getting the second order Poincaré-Pontryagin-Melnikov perturbation of some families of centers. The iterated integrals of rational functions that are necessary to reach the second order can not always be expressed in terms of elementary functions. When this happens we say that the integrals are non explicit. The calculation of these integrals involves extra difficulties compared with the calculations at the first order analysis. Hence, in general to get a higher order study may not be as easy. More concretely, since we have considered rational perturbations up to second order in $\varepsilon$, the computation of the Poincaré-Pontryagin-Melnikov functions involves the integrals

$$
\int_{-\pi}^{\theta} \frac{\sin (k \psi) \varphi(r, \psi)}{(1+r \cos \psi)^{\ell}} d \psi, \quad \int_{-\pi}^{\theta} \frac{\cos (k \psi) \varphi(r, \psi)}{(1+r \cos \psi)^{\ell}} d \psi
$$

where $\varphi$ represents either the constant 1 or the $\lambda$ or $\phi$ function, or even their corresponding primitives, being

$$
\begin{aligned}
\phi(r, \theta) & =\frac{1}{\sqrt{1-r^{2}}}\left(\theta-2 \arctan \left(\sqrt{\frac{1-r}{1+r}} \tan \left(\frac{\theta}{2}\right)\right)\right), \\
\lambda(r, \theta) & =\log (1+r \cos \theta),
\end{aligned}
$$

where $r \in(-1,1)$ and $\theta \in[-\pi, \pi]$. From the functions defined in (5) we say that the iterated integrals that appear in this paper are explicit since they can be expressed using an extension of the set of elementary functions with the functions $\phi$ and $\lambda$.

The goals of this paper are to get the shape, number and period of the hyperbolic periodic solutions bifurcating from the period annulus for some families of equations (1). We use a second order approach based on a generalization of the method introduced in [9] and improved in [33]. In the former reference the first non-zero derivative of the return map associated with orbits of a perturbed Hamiltonian system, $d H+\varepsilon \omega=0$ where $H(x, y)=x^{2}+y^{2}$, is done. In the latter one, 
the method is extended to radial Hamiltonians to study the shape of an orbit as well. A higher order study when $\omega$ is a polynomial one form is carried out in [32] because all the iterated primitives are obtained in terms of elementary functions.

The averaging theory can be an equivalent approach to obtain bifurcated periodic solutions for equations of type (1). Recent works, where higher order analysis of this theory is developed and applied to study the periodic orbits that some polynomial vector fields present, are [3, 17].

In Section 2 we study the shape, number and period of the isolated periodic solutions concerning a subclass of Abel differential equations. In Section 3, as a second application, we examine some families of planar polynomial differential equations. In Section 4 simultaneous bifurcation of limit cycles, from two continua of periodic orbits in quadratic isochronous centers, is considered. In Section 5 we summarize what the main impediments are to get higher order. Finally, in Appen$\operatorname{dix}$ A we write down the expressions and properties of the rational trigonometric integrals shared by the families in this paper, the use of which allows us to achieve the higher order studies. As we will see in the proof of the results, the integrals of Appendix A are useful for all the computations because the involved denominators are powers of polynomials of degree one in $\cos \theta$. Below we detail the contents of Sections 2, 3 and 4 .

In Section 2 we consider the differential equation

$$
\frac{d r}{d \theta}=a(\theta) r^{2}+\varepsilon \sum_{i=0}^{m} P_{i}(\theta) r^{i}+\varepsilon^{2} \sum_{i=0}^{m} Q_{i}(\theta) r^{i}
$$

where $a(\theta), P_{i}(\theta)$ and $Q_{i}(\theta)$ are analytic and $2 \pi$-periodic in $\theta \in[-\pi, \pi]$. We define the functions

$$
\begin{aligned}
F_{1}(r)= & \sum_{i=0}^{m} \int_{-\pi}^{\pi} r^{i} P_{i}(\theta)(1-r A(\theta))^{2-i} d \theta \\
F_{2}(r)= & \sum_{i=0}^{m} \int_{-\pi}^{\pi} r^{i} Q_{i}(\theta)(1-r A(\theta))^{2-i} d \theta \\
& +\sum_{i=0}^{m} \int_{-\pi}^{\pi} i r^{i-1} W_{1}(r, \theta) P_{i}(\theta)(1-r A(\theta))^{1-i} d \theta \\
& +\int_{-\pi}^{\pi} a(\theta)\left(W_{1}(r, \theta)\right)^{2}(1-r A(\theta))^{-2} d \theta,
\end{aligned}
$$

where $A(\theta)=\int_{-\pi}^{\theta} a(\psi) d \psi$ and $W_{1}(r, \theta)=\sum_{i=0}^{m} \int_{-\pi}^{\theta} r^{i} P_{i}(\psi)(1-r A(\psi))^{2-i} d \psi$.

From the above definitions we state next theorem which generalizes some of the results given by Françoise in [10].

Theorem 1. Consider the differential equation (6) and the corresponding functions given in (7) with a $2 \pi$-periodic function $A(\theta), \theta \in[-\pi, \pi]$.

(i) If $F_{1}(\rho)=0$, and $F_{1}^{\prime}(\rho) \neq 0$, then (6) has a hyperbolic $2 \pi$-periodic orbit which tends to $\rho(1-\rho A(\theta))^{-1}$ when $\varepsilon$ goes to zero, and it is written as

$$
r(\theta ; \rho, \varepsilon)=\frac{\rho}{1-\rho A(\theta)}+\varepsilon \frac{r_{1}(-\pi ; \rho)+W_{1}(\rho, \theta)}{(1-\rho A(\theta))^{2}}+O\left(\varepsilon^{2}\right)
$$

where $r_{1}(-\pi ; \rho)$ is the solution of the equation $F_{2}(\rho)=0$. 
(ii) When $F_{1}=0$, if $F_{2}(\rho)=0$ and $F_{2}^{\prime}(\rho) \neq 0$ then (6) has a hyperbolic $2 \pi$ periodic orbit that tends to $\rho(1-\rho A(\theta))^{-1}$ when $\varepsilon$ goes to zero.

We observe that, from the statement $(i)$ of above theorem, shape up to first order term in $\varepsilon$ of a limit cycle needs a second order study to be determined completely. This can be seen in all the examples of next sections. Moreover, the functions $F_{1}$ and $F_{2}$ defined in $(7)$ are the first and second Poincaré-Pontryagin-Melnikov functions for equation (6). As an application, next proposition bounds, up to second order study, the number of periodic solutions of a polynomial perturbation of equation (6) for a class of Abel equations with $a(\theta)=\sin \theta$. The first order analysis for a restricted perturbation is shown in the previous works $[1,13]$, when $n$ is arbitrary.

Proposition 2. Consider the differential equation (6) with $m=3, a(\theta)=\sin \theta$, and $P_{i}, Q_{i}$ trigonometric polynomials of degree $n$, for $i=0, \ldots, 3$. The maximum number of hyperbolic periodic solutions, for $\varepsilon$ small enough and $1 \leq n \leq 6$, is $n+3$ and $\max \{n+3,2 n+4\}$ using first and second order studies, respectively. When $n=0$ both maximal numbers are 3 . Moreover, these upper bounds are sharp.

This result shows how the number of hyperbolic periodic solutions increases with a second order study, at least for the first values of $n$, when the first order vanishes identically. In fact this number is almost doubled using perturbations of second order.

In Section 2 we also show that for concrete perturbations another upper bounds are obtained. In Proposition 8 we prove this fact and Proposition 10 gives an example exhibiting the two periodic solutions provided by former result, when a concrete perturbation with trigonometric polynomials of degree one is considered.

The second application concerning the integrals given in Section A is done in Section 3. It deals with planar differential equations that, after a change of variables if necessary, are written as

$$
\left\{\begin{array}{l}
\dot{x}=-y+\varepsilon P_{1}(x, y)+\varepsilon^{2} P_{2}(x, y) \\
\dot{y}=x+\varepsilon Q_{1}(x, y)+\varepsilon^{2} Q_{2}(x, y)
\end{array}\right.
$$

where $P_{i}(x, y)$ and $Q_{i}(x, y)$ are analytic functions. This is the case of second order approximation of the perturbed Hamiltonian radial differential equation. In short, we consider the differential equation

$$
\left\{\begin{aligned}
x^{\prime} & =-\frac{\partial H}{\partial y}+\varepsilon P(x, y, \varepsilon), \\
y^{\prime} & =\frac{\partial H}{\partial x}+\varepsilon Q(x, y, \varepsilon),
\end{aligned}\right.
$$

when $H$ is an analytic function that, in polar coordinates, is written as $H=H(r)$. The last system, after a rescaling of time, is written as (8) because $\partial H / \partial y=$ $y r^{-1} d H / d r$ and $\partial H / \partial x=x r^{-1} d H / d r$. This case is also studied in [32].

All the families that are considered in this second application can be transformed into (8) where the perturbations of the linear center are rational functions. In fact, all the denominators in polar coordinates are powers of a polynomial of degree one in $\cos \theta$.

The first family of planar differential equations that we consider is studied in Section 3.1. It is the polynomial perturbation of a linear center with a line of 
singular points that does not pass through the center. It is not restrictive to consider only the family given by

$$
\left\{\begin{array}{l}
x^{\prime}=-y(1+x)+\varepsilon P_{1}(x, y)+\varepsilon^{2} P_{2}(x, y) \\
y^{\prime}=x(1+x)+\varepsilon Q_{1}(x, y)+\varepsilon^{2} Q_{2}(x, y)
\end{array}\right.
$$

Here $P_{i}(x, y)$ and $Q_{i}(x, y), i=1,2$, are polynomials of degree $n$. Note that this differential equation is written as $(8)$ after a $(1+x)$-rescaling of time. The number of periodic orbits of (10), using the first Poincaré-Pontryagin-Melnikov function, is done in [26], where $n$ hyperbolic periodic orbits are found. An upper bound for this number is given in [28], using a second order study, when the first order vanishes identically. This upper bound is too high for concrete values of $n$ as it can be seen in Proposition 3. In [27] an example of a quadratic perturbation with three limit cycles is provided up to first order study.

Additionally, the shape and the period of a periodic orbit for a particular perturbation is obtained in Proposition 12. A third order study for quadratic perturbations is carried out in [2] using Cartesian coordinates.

Proposition 3. System (10) has at least $\max (2 n-2, n)$ hyperbolic periodic orbits when $n \leq 10$.

The second family of planar differential equations that we consider is the polynomial perturbation of some isochronous centers, see Section 3.2. We write this family as

$$
\left\{\begin{array}{l}
\dot{x}=P_{0}(x, y)+\varepsilon P_{1}(x, y)+\varepsilon^{2} P_{2}(x, y) \\
\dot{y}=Q_{0}(x, y)+\varepsilon Q_{1}(x, y)+\varepsilon^{2} Q_{2}(x, y)
\end{array}\right.
$$

where $P_{i}, Q_{i}, i=1,2$, are polynomials. When $\varepsilon=0$, we remember that the origin of system (11) is an isochronous center when there is a change of variables, $\varphi(x, y)$, that moves it to (8). This change is called its linearization. Note that, as the time of both equations is the same, the period of the periodic solutions also coincides. Only the case when the linearization and its inverse are polynomial is considered in [32].

Quadratic isochronous centers are classified in [29] in four families called $S_{1}$, $S_{2}, S_{3}$ and $S_{4}$. A unified proof of the isochronicity property, as well as their linearization, can be found in [30]. The expressions of the vector fields $S_{i}$ that we use in this paper come from [4]. Except for $S_{4}$, the function $\varphi$ is a birational one. Hence, the perturbation of (8) only is rational for $S_{1}, S_{2}$ and $S_{3}$ and the integrals of Appendix A are useful to study (11). These three families are written, respectively, as

$$
\left\{\begin{array} { l } 
{ \dot { x } = - y + x y , } \\
{ \dot { y } = x - \frac { 1 } { 2 } x ^ { 2 } + \frac { 1 } { 2 } y ^ { 2 } , } \\
{ \dot { y } = x - y ^ { 2 } , }
\end{array} \quad \left\{\begin{array}{l}
\dot{x}=-y(1+x), \\
\dot{y}=-x\left(1-\frac{16}{3} y\right) .
\end{array}\right.\right.
$$

Figure 1 shows the phase portraits of these families and only the first one presents two continua of periodic orbits. The first order perturbation problem for the above families is considered in [6]. Next result revisits this study and goes on to the second order quadratic perturbation. Concerning the simultaneousness of limit cycles we have next definition. 

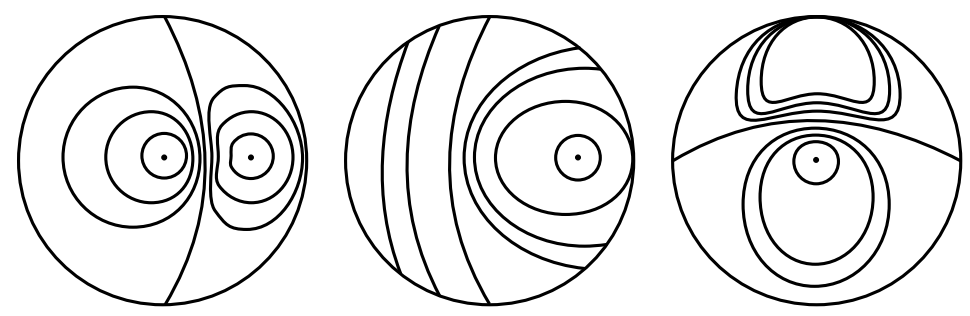

Figure 1. Phase portraits in the Poincaré sphere of systems $S_{1}, S_{2}$ and $S_{3}$, respectively.

Definition 4. For a planar vector field with two foci, $f_{k}, k=1,2$, we will say that the configuration of limit cycles $(i, j), i \geq 0, j \geq 0$, is realizable if exactly $i$ (respectively $j$ ) limit cycles surround $f_{1}$ (respectively $f_{2}$ ).

Theorem 5. Consider a perturbed system as (11) for each differential equation given in (12). Then each family, using up to a second order study in $\varepsilon$, has no more than two hyperbolic periodic orbits under quadratic perturbations and this number is sharp. Moreover, the $S_{1}$ family presents two limit cycles in realizable configuration $(2,0),(1,1)$ or $(0,2)$.

The proof of this theorem follows from Propositions 13 and 18. The latter proposition deals with the simultaneous bifurcation of limit cycles from the two centers of $S_{1}$, using first and second order perturbations. There are not to many studies of simultaneous bifurcation from more than one continuum of periodic orbits, some works related on simultaneity on quadratic vector fields are $[5,6,11$, 23].

As a final application of the integrals of Appendix A we obtain the shape of periodic orbits that bifurcate, for some concrete quadratic perturbations, from the isochronous centers $S_{1}, S_{2}$ and $S_{3}$. This is addressed in Propositions 15, 16 and 17 , respectively.

The computations in this paper are made with the algebraic manipulator MAPLE and we note that the study of the second order is hard to do for polynomials of higher order.

\section{Perturbation of Abel equations}

In this section we are concerned with both the number and shape of the hyperbolic periodic solutions, bifurcating from the period annulus around $r=0$, of the perturbed equation (6). First we provide the expressions of $r_{i}(\theta ; \rho), i=1,2$, for the solutions of (6) (see Proposition 6). Second we prove Theorem 1, which gives conditions for the existence of hyperbolic periodic solutions bifurcating from the period annulus of equation (6). In this result, additionally, the shape of these solutions is also given. Next we prove Proposition 2, where the number of periodic orbits bifurcating up to second order analysis for a class of polynomial trigonometric Abel equations is done. Moreover, Proposition 7 extends the results for the class of Abel equation considered in $[1,13]$ up to a second order study. Finally, we relate the results obtained in Propositions 2 and 7. At the end of this section, we apply these results to obtain the shape of a hyperbolic periodic orbit in a particular equation (see Proposition 8). 
Proposition 6. Consider the differential equation (6) and let $r(\theta ; \rho, \varepsilon)$ be a solution so that $r(-\pi ; \rho, 0)=\rho$. Then the first and second terms of the series in $\varepsilon$ of (2) are

$$
r_{i}(\theta ; \rho)=(1-\rho A(\theta))^{-2}\left(r_{i}(-\pi ; \rho)+\int_{-\pi}^{\theta} w_{i}(\rho, \psi) d \psi\right),
$$

for $i=1,2$, where

$$
\begin{aligned}
w_{1}(\rho, \theta)= & \sum_{i=0}^{m} \rho^{i} P_{i}(\theta)(1-\rho A(\theta))^{2-i} \\
w_{2}(\rho, \theta)= & \sum_{i=0}^{m} \rho^{i} Q_{i}(\theta)(1-\rho A(\theta))^{2-i} \\
& +r_{1}(\theta ; \rho)(1-\rho A(\theta))^{2}\left(a(\theta) r_{1}(\theta ; \rho)+\sum_{i=0}^{m} i \rho^{i-1} P_{i}(\theta)(1-\rho A(\theta))^{1-i}\right) .
\end{aligned}
$$

Proof. The expression of the statement follows directly if we substitute the series expansion of $r(\theta ; \rho, \varepsilon)$ in equation (6) and by using a proof by induction on $m$.

From the above expressions of $r_{i}$ we get the proof of Theorem 1 as follows.

Proof of Theorem 1. Proposition 6 gives the first and second terms of the series expansion in $\varepsilon$ of every solution of equation (6). Then, we impose that this solution is periodic for every $\varepsilon$; that is $r(\pi ; \rho, \varepsilon)=r(-\pi ; \rho, \varepsilon)$. The necessary condition up to first order is $F_{1}(\rho)=0$, or equivalently $r_{1}(\pi ; \rho)=r_{1}(-\pi ; \rho)$. From the Implicit Function Theorem, the simple zeros of $F_{1}(\rho)$ provide hyperbolic periodic solutions of (6), for $\varepsilon$ small enough. Nevertheless it is not possible to get the value $r_{1}(-\pi ; \rho)$ at this step. This value is obtained from $F_{2}(\rho)=0$, or equivalently from $r_{2}(\pi ; \rho)=r_{2}(-\pi ; \rho)$. That is, forcing the solution to be periodic up to second order in $\varepsilon$. This proves statement (i). Statement (ii) follows analogously assuming that $F_{1}(r) \equiv 0$.

It is worth mentioning that when we look for the shape of a periodic orbit, the value of $r_{i}(-\pi ; \rho)$ is fixed from the study of the return map given up to order $i+1$.

As an application of former results together with the integrals of Appendix A, we study lower bounds for the number of hyperbolic periodic solutions of the perturbed equation (6) for an Abel subclass. We use first and second order approaches, with $a(\theta)=\sin \theta$ and the trigonometric polynomials of degree $n, P_{i, n}(\theta)$ and $Q_{i, n}(\theta)$. In this case the interval of definition is $(-1 / 2, \infty)$. This study is done for $n \leq 6$ and $n \leq 9$ in Proposition 2 and 7, respectively. The first order analysis for the latter result agrees with the previous works $[1,13]$ where an arbitrary $n$ is considered. Hence we concentrate our attention to the second order study but, due to the computational difficulties, we restrict our analysis to lower values of the degree $n$. Despite the similarity of the proofs of both results, there are some interesting differences that we would discuss after proving both propositions.

Proposition 7. Consider the differential equation (6) with $m=3, a(\theta)=\sin \theta$, and $P_{i}, Q_{i}$ trigonometric polynomials of degree $n$, for $i=0, \ldots, 3$. The maximum number of hyperbolic periodic solutions, for $\varepsilon$ small enough, $n \leq 9$ and $P_{i}=Q_{i}=0$ for $i=0,1$, is $n+1$ and $\max \{n+1,2 n\}$ using first and second order studies, respectively. Moreover these upper bounds are sharp. 
Proof. Consider a solution of the differential equation (6) with $a(\theta)=\sin \theta$ and the initial condition $r(-\pi ; \rho)=\rho$. When $\varepsilon=0$, this solution is written as

$$
r(\theta ; \rho)=\frac{\rho}{1+\rho+\rho \cos \theta}
$$

and it is well-defined for $\rho \in(-1 / 2, \infty)$. Consequently every solution is $2 \pi$ periodic. Taking $a(\theta)=\sin \theta$ and $A(\theta)=-1-\cos \theta$, the functions $F_{1}(\rho)$ and $F_{2}(\rho)$ follow from (7). These functions are computed, for $n \leq 9$, changing $\rho$ by $r /(1-r)$ and using the expressions of the integrals of Appendix A. For $n \geq 1$, the change of variables $r=\sqrt{1-s^{2}}$ gives $F_{i}(\rho)=\widetilde{F}_{i}(s)\left(1-s^{2}\right) /\left(1-\sqrt{1-s^{2}}\right)^{2}$ for $i=1,2$, where

$$
\begin{aligned}
& \widetilde{F}_{1}(s)=\frac{\sqrt{1-s^{2}}}{s(1+s)^{\left[\frac{n}{2}\right]}} U_{1,\left[\frac{n}{2}\right]}(s)+\frac{1}{s(1+s)^{\left[\frac{n-1}{2}\right]} V_{1,\left[\frac{n+1}{2}\right]}(s),} \\
& \widetilde{F}_{2}(s)=\frac{1}{s(1+s)^{n-1}}\left(U_{2, n}(s)+V_{2, n-1}(s) \sqrt{1-s^{2}}\right) .
\end{aligned}
$$

Here $U_{i, j}$ and $V_{i, j}$ are polynomials of degree $j$ in $s$ and of degree $i$ in the parameters of the perturbation.

The functions $\widetilde{F}_{1}$ and $\widetilde{F}_{2}$ have at most $n+1$ and $2 n$ zeros, respectively. This is due to the fact that $\widetilde{F}_{i}(s)=0, i=1,2$, can be transformed, by squaring, to a polynomial of degrees $n+1$ and $2 n$, respectively. Choosing, in a convenient way, the polynomials of the perturbation we get $U_{i, j}$ and $V_{i, j}$ with arbitrary coefficients. Then, these bounds are sharp. The proof ends because the simple zeros of these functions, by applying Theorem 1, give rise to hyperbolic periodic orbits.

The case $n=0$ requires a separate study to get the analogous expressions (13).

Proof of Proposition 2. The proof follows as in Proposition 7. Hence we only detail the differences.

When $n \geq 1$, the functions $F_{1}$ and $F_{2}$ given in (7) write, with the change of variables $\rho=r /(1-r)$ and $r=\sqrt{1-s^{2}}$, as $F_{i}(\rho)=\widetilde{F}_{i}(s) /\left(1-\sqrt{1-s^{2}}\right)^{2}$ for $i=1,2$, with

$$
\begin{aligned}
& \widetilde{F}_{1}(s)=\frac{1+s}{s}\left(\frac{\sqrt{1-s^{2}}}{(1+s)^{\left[\frac{n}{2}\right]}} U_{1,\left[\frac{n}{2}\right]+1}(s)+\frac{1}{(1+s)^{\left[\frac{n-1}{2}\right]}} V_{1,\left[\frac{n+1}{2}\right]+1}(s)\right), \\
& \widetilde{F}_{2}(s)=\frac{1}{s(1+s)^{n-1}}\left(U_{2, n+2}(s)+V_{2, n+1}(s) \sqrt{1-s^{2}}\right) .
\end{aligned}
$$

Here $U_{i, j}$ and $V_{i, j}$ are polynomials of degree $j$ in $s$ and of degree $i$ in the parameters of the perturbation and the bounds for the number of zeros are those given in the statement. The expression of $\widetilde{F}_{1}(s)$ comes also from the expression (13) by adding the perturbation terms $P_{0}(\theta)$ and $P_{1}(\theta)$. In fact this perturbation contribute to $\widetilde{F}_{1}(s)$ only adding a term of the form $a_{0}+a_{1} s^{2}+a_{2} \sqrt{1-s^{2}}$.

All the coefficients of $U_{1, j}$ and $V_{1, j}$, through a linear change of variables in the parameter space, can be chosen independently. This is not the case for all the coefficients of $U_{2, j}$ and $V_{2, j}$ because there is exactly one of them that depends nonlinearly on the other coefficients. This can be taken as the leading coefficient of $U_{2, j}$ and different from zero. Hence, the sharpness of the upper bounds are guaranteed. 
The case $n=0$ requires a separate study to get the analogous expressions (14). In this case only three zeros appear in both functions.

Notice that although we prove the expressions of $F_{2}$ given in (13) and (14) for every $n$, the main hurdle is to prove the independence of the coefficients of the polynomials $U_{2, n}$ and $V_{2, n-1}$. We remark that, as we mentioned in the above proof, to get the independence is a more delicate problem for the general perturbation. Also we observe that $\left(1-s^{2}\right)$ is a common factor for the functions $F_{i}, i=1,2$ that appear in the proof of Proposition 7. This implies that the degrees of the polynomials $U_{i, j}$ and $V_{i, j}$ given in (13) decrease by one and two with respect to the ones in (14), for $F_{1}$ and $F_{2}$ respectively. Hence the number of zeros also decrease in two and four for the first and second order study, respectively.

Proposition 7 ensures that, for $n=1$, this procedure generates at most two hyperbolic periodic orbits. We illustrate this fact by showing their shape, for a concrete perturbation. It can be seen in the proof that the first order can be determined only up to a second order study.

Proposition 8. The differential equation

$$
\frac{d r}{d \theta}=\sin \theta r^{2}+\varepsilon\left(\left(-\frac{1}{6}+2 \cos \theta+\sin \theta\right) r^{2}+\left(1+\frac{7}{3} \cos \theta\right) r^{3}\right)-\varepsilon^{2} \cos \theta r^{2}
$$

has two hyperbolic periodic solutions. The first terms of their power series expansion in $\varepsilon$ are

$$
\begin{aligned}
r(\theta ; 3 / 2, \varepsilon)= & \frac{3}{5+3 \cos \theta}+\varepsilon \frac{-305+234 \phi(3 / 5, \theta)+270 \sin \theta-135 \cos \theta}{15(5+3 \cos \theta)^{2}}+O\left(\varepsilon^{2}\right), \\
r(\theta ; 9 / 32, \varepsilon)= & \frac{9}{41+9 \cos \theta} \\
& +\varepsilon \frac{20213+21060 \phi(9 / 41, \theta)+19926 \sin \theta-9963 \cos \theta}{123(41+9 \cos \theta)^{2}}+O\left(\varepsilon^{2}\right),
\end{aligned}
$$

where $\phi$ is defined in (5).

Proof. Let us consider the differential equation (15) with the initial condition $r(-\pi ; \rho, 0)=\rho$ for $\rho \in(-1 / 2, \infty)$. From Theorem 1 the hyperbolic periodic orbits bifurcate from

$$
r(\theta ; \rho, 0)=\frac{\rho}{1+\rho+\rho \cos \theta} .
$$

Using the change of variables $\rho=s /(1-s)$, the functions in $(7)$, from the expressions of the integrals of Appendix A, write as $F_{i}(\rho)=2 \pi s^{2} \widetilde{F}_{i}(s) /(1-s)^{2}$ for $i=1,2$ where

$$
\widetilde{F}_{1}(s)=\frac{13}{6} s^{2}+\left(-\frac{7}{3} s^{2}+s^{3}\right) \frac{1}{\sqrt{1-s^{2}}} .
$$

This function has two simple zeros at $s_{1}=3 / 5\left(\widetilde{F}_{1}^{\prime}(3 / 5)=-9 / 32\right)$ and $s_{2}=9 / 41$ $\left(\widetilde{F}_{1}^{\prime}(9 / 41)=81 / 3200\right)$ and they correspond with the initial values $\rho_{1}=3 / 2$ and $\rho_{2}=9 / 32$. Furthermore,

$$
\begin{aligned}
r_{1}\left(\theta ; \rho_{i}\right)=r_{1}\left(-\pi ; \rho_{i}\right)\left(1-s_{i}\right)^{2} & +\frac{\left(3 s_{i}-7\right) s_{i}^{2}}{3}\left(\pi\left(1-s_{i}^{2}\right)^{-\frac{1}{2}}-\phi\left(s_{i}, \theta\right)\right) \\
& +2 s_{i}^{2} \sin \theta-s_{i}^{2} \cos \theta+\frac{(-6+13 \pi) s_{i}^{2}}{6}
\end{aligned}
$$


for $i=1,2$ and

$$
\widetilde{F}_{2}\left(s_{1}\right)=-\frac{51}{400}-\frac{9}{200} r_{1}\left(-\pi ; \rho_{1}\right), \quad \widetilde{F}_{2}\left(s_{2}\right)=-\frac{621}{168100}+\frac{648}{42025} r_{1}\left(-\pi ; \rho_{2}\right) .
$$

We obtain the values $r_{1}\left(-\pi ; \rho_{1}\right)=-17 / 6$ and $r_{1}\left(-\pi ; \rho_{2}\right)=23 / 96$ as solutions of $\widetilde{F}_{2}\left(s_{i}\right)=0$ for $i=1,2$. Hence the statement follows by substituting these values in equation (16).

Last result provides the first order approximations in $\varepsilon$ of $r\left(-\pi ; \rho_{i}, \varepsilon\right), i=1,2$, that is, their linear approximations. Figure 2 shows the tangent lines (solid line) together with the numerical approximations (dotted line) for $\varepsilon \in[-0.177,0.363]$. Both solutions, for $\varepsilon=0.1$, are plotted in Figure 3 .

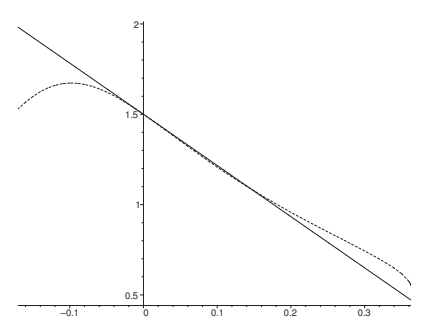

(a) $\rho_{1}=3 / 2$

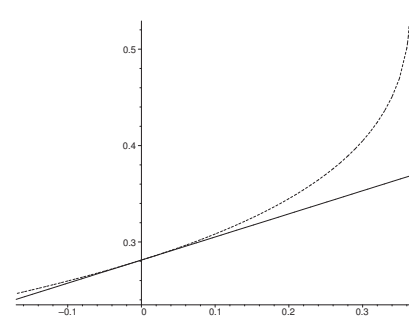

(b) $\rho_{2}=9 / 32$

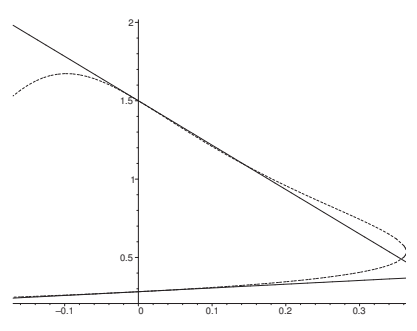

(c)

FiguRE 2. First order (solid line) and numerical (dotted line) approximations in $\varepsilon \in[-0.177,0.363]$ of $r\left(-\pi ; \rho_{i}, \varepsilon\right), i=1,2$, in Proposition 8. Both approximations are plotted together in (c).

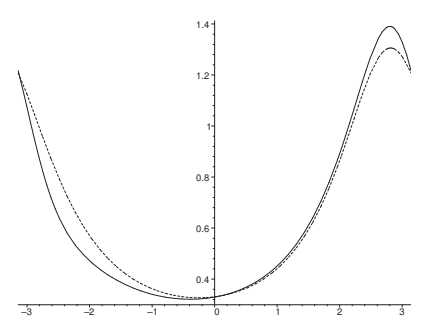

(a) $\rho_{1}=3 / 2$

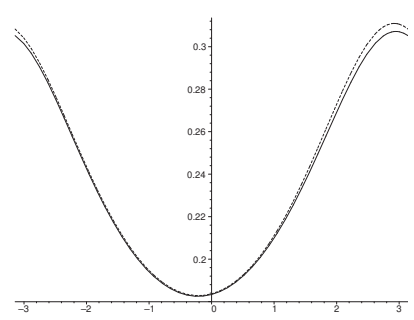

(b) $\rho_{2}=9 / 32$

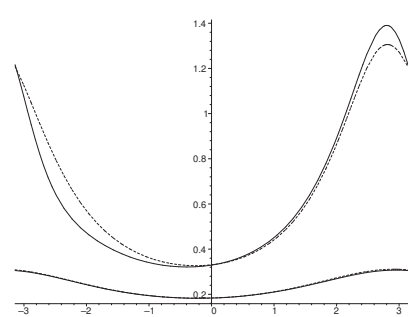

(c)

FiguRE 3. First order (solid line) and numerical (dotted line) approximations of the periodic solutions in Proposition $8, r\left(\theta ; \rho_{i}, 0.1\right)$ for $i=1,2$ and $\theta \in[-\pi, \pi]$. Both solutions are plotted in (c).

To finish this section, we would like to remark that the main obstacle for going further in the power series in $\varepsilon$ is the need to obtain the concrete values of $r_{j}\left(-\pi ; \rho_{i}\right)$ for $i=1,2$ and $j \geq 2$. This implies getting iterated primitives of expressions involving the functions $\phi$ and $\lambda$.

\section{Perturbation of Planar polynomial Differential Systems}

Let us assume that in equation (9) we have

$$
P(x, y, \varepsilon)=P_{1}(x, y)+\varepsilon P_{2}(x, y), \quad Q(x, y, \varepsilon)=Q_{1}(x, y)+\varepsilon Q_{2}(x, y),
$$


where $P_{i}$ and $Q_{i}$ are analytic functions. Hence, in polar coordinates given by $(x, y)=(r \cos \theta, r \sin \theta)$, equation (9) is written as the one-form

$$
r d r+\varepsilon\left(\alpha_{1}(r, \theta) d r+\beta_{1}(r, \theta) d \theta\right)+\varepsilon^{2}\left(\alpha_{2}(r, \theta) d r+\beta_{2}(r, \theta) d \theta\right)=0,
$$

where

$$
\begin{aligned}
\alpha_{i}(r, \theta) & =\cos \theta Q_{i}(r \cos \theta, r \sin \theta)-\sin \theta P_{i}(r \cos \theta, r \sin \theta), \\
\beta_{i}(r, \theta) & =-r \cos \theta P_{i}(r \cos \theta, r \sin \theta)-r \sin \theta Q_{i}(r \cos \theta, r \sin \theta)
\end{aligned}
$$

are $2 \pi$-periodic functions in $\theta$. For equation (17), following [32], we define

$$
\begin{aligned}
F_{1}(r) & =-\frac{1}{2 \pi} \int_{-\pi}^{\pi} \beta_{1}(r, \psi) d \psi \\
G_{1}(r, \theta) & =-F_{1}(r) \theta-\int_{-\pi}^{\theta} \beta_{1}(r, \psi) d \psi \\
F_{2}(r) & =-\frac{1}{2 \pi} \int_{-\pi}^{\pi}\left(\beta_{2}(r, \psi)-\beta_{1}(r, \psi)\left(\alpha_{1}(r, \psi)+\frac{\partial G_{1}(r, \psi)}{\partial r}\right)\right) d \psi
\end{aligned}
$$

where $\theta \in[-\pi, \pi]$. Next result provides the first terms of the power series expansion (2) of every periodic solution of (17) so that $r(-\pi ; \rho, 0)=\rho$. Even assuming that $r_{i}(\theta)$ depends on $\rho$, that is $r_{i}(\theta)=r_{i}(\theta ; \rho)$, to simplify the reading, we do not make it explicit except if necessary. Here $r_{0}(\theta)=\rho$, for all $\theta \in[-\pi, \pi]$, because the unperturbed part of (17) does not depend on $\theta$.

Theorem 9 ([32]). Let $r(\theta ; \rho, \varepsilon)$ be the solution of the initial value problem given by equation (17) with $r(-\pi ; \rho, 0)=\rho$.

(i) If $F_{1}(\rho)=0$ and $F_{1}^{\prime}(\rho) \neq 0$, then equation (17) has a hyperbolic periodic orbit that is written as

$$
r(\theta ; \rho, \varepsilon)=\rho+\varepsilon\left(r_{1}(-\pi)+\rho^{-1}\left(F_{1}(\rho) \theta+G_{1}(\rho, \theta)-G_{1}(\rho,-\pi)\right)\right)+O\left(\varepsilon^{2}\right),
$$

where

$$
r_{1}(-\pi)=-\frac{F_{2}(\rho)}{F_{1}^{\prime}(\rho)}-\frac{1}{2 \pi \rho} \int_{-\pi}^{\pi}\left(G_{1}(\rho, \theta)-G_{1}(\rho,-\pi)\right) d \theta
$$

Also, its period writes as

$$
T(\varepsilon ; \rho)=2 \pi-\frac{\varepsilon}{\rho} \int_{-\pi}^{\pi} \alpha_{1}(\rho, \theta) d \theta+O\left(\varepsilon^{2}\right) .
$$

(ii) If $F_{1}=0, F_{2}(\rho)=0$ and $F_{2}^{\prime}(\rho) \neq 0$, then equation (17) has a hyperbolic periodic orbit bifurcating from $r(\theta ; \rho, \varepsilon)=\rho+O(\varepsilon)$.

We observe that the functions $F_{1}$ and $F_{2}$ are the first and second PoincaréPontryagin-Melnikov functions for equation (17). Moreover, the initial condition $r_{1}(-\pi)$ that determines completely the shape up to first order depends on the bifurcation of second order.

The last result gives, not only the shape and period but the conditions for the existence of periodic orbits bifurcating from a center using first and second order analysis in $\varepsilon$. In the following sections we apply it to various families. 
3.1. Linear centers with a line of singular points. In this section we consider polynomial perturbations of a linear center with a straight line of singular points that does not pass through the center. These differential systems can be written as (10) and, after a rescaling of time, they become

$$
\left\{\begin{array}{l}
\dot{x}=-y+\frac{\varepsilon P_{1}(x, y)+\varepsilon^{2} P_{2}(x, y)}{1+x}, \\
\dot{y}=x+\frac{\varepsilon Q_{1}(x, y)+\varepsilon^{2} Q_{2}(x, y)}{1+x},
\end{array}\right.
$$

when $x \neq-1$. Changing to the usual polar coordinates, the last system is expressed as (17) where $\alpha_{i}$ and $\beta_{i}$ are rational functions in $(r, \cos \theta, \sin \theta)$ with denominator powers of $(1+r \cos \theta)$. We consider this perturbed system when $\theta \in[-\pi, \pi]$. After these transformations, we study only the periodic orbits bifurcating from circles in the disk $\left\{(x, y) \in \mathbb{R}^{2}: x^{2}+y^{2}<1\right\}$.

The shape of the hyperbolic periodic orbits of (10) can be obtained by applying Theorem 9 to (19) written in the form (17). Nevertheless, since there is a rescaling of time, the period of these periodic orbits cannot be obtained directly from Theorem 9. Proposition 12 illustrates this fact by obtaining the period as well as their shape for a concrete perturbation.

Concerning the number of periodic orbits of (10), in Proposition 3 we obtain at least $2 n-2$ hyperbolic periodic orbits from a second order study for $n \leq 10$. The first order analysis done in [26] shows that the number of periodic solutions is at most $n$ and this lower bound is sharp. A second order study is performed in [28] for the particular case $P_{1}(0,0)=Q_{1}(0,0)=0$ and $P_{2}=Q_{2}=0$. For the quadratic family, a third order study is done in [2] and provides three hyperbolic periodic orbits. That is, one more than the previous studies.

We remark that we have not been able to extend Proposition 3 for all $n$, because the size of the functions involved in the computations increase very fast with $n$. Even if we obtain these functions, the main obstruction to going further in $n$, as can be seen in the proof, is knowing how to guarantee the independence of their coefficients because they depend quadratically on the perturbation parameters.

Proof of Proposition 3. We have done the proof for each $n \leq 10$ doing all the computations explicitly. We present them in a unified way.

From the integrals given in Appendix A, (18) gives

$$
\begin{aligned}
& F_{1}(r)=\frac{r^{2}}{1+\sqrt{1-r^{2}}}\left(\frac{U_{1,\left[\frac{n}{2}\right]}\left(r^{2}\right)}{\sqrt{1-r^{2}}}+V_{1,\left[\frac{n-1}{2}\right]}\left(r^{2}\right)\right), \\
& F_{2}(r)=\frac{r^{2}}{1+\sqrt{1-r^{2}}}\left(\frac{U_{2, \max \left(n-1,\left[\frac{n}{2}\right]\right)}\left(r^{2}\right)}{\sqrt{1-r^{2}}}+V_{2, \max \left(n-2,\left[\frac{n-1}{2}\right]\right)}\left(r^{2}\right)\right) .
\end{aligned}
$$

Here $U_{i, j}, V_{i, j}$ are polynomials of degree $j$ on $r^{2}$ and degree $i$ on the coefficients of the perturbation; [.] denotes the integer part function. With the change of variables $r=\sqrt{1-s^{2}}$ we get

$$
\widehat{F}_{1}(s)=\frac{1}{s} \widehat{U}_{1, n}(s), \quad \widehat{F}_{2}(s)=\frac{s-1}{s} \widehat{U}_{2,2 n-2}(s),
$$

where $\widehat{U}_{i, j}(s)$ are polynomials of degree $j$. Note that $\widehat{F}_{2}(s)$ makes sense when $\widehat{F}_{1}=0$. This provides the bound of the statement. 
Let us write $\widehat{U}_{2,2 n-2}(s)=\sum_{i=0}^{2 n-2} u_{i} s^{i}$. Here, as we have mentioned before, the parameters that appear in coefficients, $u_{i}$, depend quadratically on the coefficients of the perturbation of system (10). Additionally, there is a choice of non-vanishing $2 n-1$ parameters in the perturbation, that we denote them by $\gamma_{i}, i=0, \ldots, 2 n-2$, with $u_{i}=u_{i}\left(\gamma_{0}, \ldots, \gamma_{2 n-2}\right)$ for all $i$, such that $u_{i}\left(\gamma_{0}, \ldots, \gamma_{2 n-2}\right)$ is a polynomial of degree one in $\gamma_{i}$ for all $i$. The system $v_{i}=u_{i}\left(\alpha_{0}, \ldots, \alpha_{2 n-2}\right)$, for $i=0, \ldots, 2 n-2$, with $v_{0}=1$ has only one solution. Hence, we can write $\widehat{U}_{2,2 n-2}(s)=\sum_{i=0}^{2 n-2} v_{i} s^{i}$, with $v_{i}$ the new arbitrary parameters. Then, the polynomial $\widehat{U}_{2,2 n-2}(s)$ has as many zeros as its degree and, moreover, placed where you want. The sharpness of the bound follows if we study the zeros of $\widehat{F}_{1}(s)$ in an equivalent way. In this case the linear dependence of the coefficients on the perturbation simplifies the computations.

The proof ends by applying Theorem 9 .

In the last proof, an alternative study for the number of zeros of $F_{i}(r)$, defined in (20), can also be made following [12]. In this reference, functions of type (20) have been considered and it is proved that they have as many zeros as the number of monomials minus one. That is $(\max (n-1,[n / 2])+1)+$ $(\max (n-2,[(n-1) / 2])+1)-1=\max (2 n-2, n)$. Another approach, which is only useful for small values of $n$, is to get a concrete perturbation in (10) requiring that the corresponding $\widehat{U}_{2,2 n-2}(s)$ has zeros at, for example, $s_{i}=1 /(i+1)$, $i=1, \ldots, 2 n-2$. Next proposition illustrates this procedure for a perturbation of degree four.

Proposition 10. System

$$
\left\{\begin{array}{l}
x^{\prime}=-y(1+x)-\varepsilon \frac{\left(255+255 x-256 y^{2}\right) y^{2}}{80}+\varepsilon^{2} \frac{720+719 x^{3}+29996 x^{2} y^{2}}{720}, \\
y^{\prime}=x(1+x)-\varepsilon \frac{(16 x-5 y) y^{3}}{5}+\varepsilon^{2} \frac{\left(30318 x^{2}-6769 y^{2}\right) y}{720}
\end{array}\right.
$$

satisfies that $F_{1}=0$ and $F_{2}$ has six simple zeros.

Proof. Following the steps of the proof of Proposition 3, we succeed in getting that, in $(20)$ and $(21), F_{1}(r) \equiv 0$ and $\widehat{F}_{2}(s)=(s-1)(7 s-1)(6 s-1)(5 s-1)(4 s-$ $1)(3 s-1)(2 s-1) /(720 s)$.

Remark 11. We note that, for $n \geq 2$, the previous upper bound $\max (2 n-2, n)$ is in fact $2 n-2$. This number is one less than the bound for the number of zeros of $F_{2}$ given in [28]. This improvement is a consequence of the structure of $F_{2}$, see (20). In fact, the polynomial $\widehat{U}_{2,2 n-2}(s)$ has an extra factor $(s-1)$ and, consequently, the bound decreases in one. Moreover the perturbation considered in [28] satisfies certain restrictions.

Next example shows how to obtain the shape, up to first order in $\varepsilon$, when a concrete perturbation of degree one for family (10) is considered.

Proposition 12. Let us consider the perturbed system

$$
\left\{\begin{array}{l}
x^{\prime}=-y(1+x)-\varepsilon(4-y) \\
y^{\prime}=x(1+x)+\varepsilon(4-5 y) .
\end{array}\right.
$$


Then, it has a hyperbolic periodic orbit so that its radius is written as

$$
\begin{aligned}
r\left(\theta ; \frac{3}{5}, \varepsilon\right)=\frac{3}{5}+\varepsilon & -\frac{25}{3}+25 \log 2-10 \log 3+5 \sin \theta-\cos \theta \\
& \left.-12 \phi\left(\frac{3}{5}, \theta\right)-5 \log (5+3 \cos \theta)\right)+O\left(\varepsilon^{2}\right),
\end{aligned}
$$

with period

$$
T\left(\varepsilon ; \frac{3}{5}\right)=\frac{5 \pi}{2}+\frac{625 \pi}{96} \varepsilon+O\left(\varepsilon^{2}\right) .
$$

Here the function $\phi$ is defined in (5).

Proof. System (22), in polar coordinates, is equivalent to (17) with

$$
\begin{aligned}
& \alpha_{1}(r, \theta)=\frac{-r+8 \sin \theta+8 \cos \theta-5 r \sin (2 \theta)+r \cos (2 \theta)}{2(1+r \cos \theta)}, \\
& \beta_{1}(r, \theta)=\frac{5 r^{2}-8 r \sin \theta+8 r \cos \theta-r^{2} \sin (2 \theta)-5 r^{2} \cos (2 \theta)}{2(1+r \cos \theta)}
\end{aligned}
$$

and $\alpha_{2}=\beta_{2}=0$. Here $0<r<1$. Then, using the expressions of the integrals of Appendix A, the functions given in (18) write as

$$
\begin{aligned}
F_{1}(r)= & -9+\frac{9-5 r^{2}}{\sqrt{1-r^{2}}}, \\
G_{1}(r, \theta)= & -3 \log \left(\frac{1+r \cos \theta}{1-r}\right)+\left(9-5 r^{2}\right)\left(\frac{\pi}{\sqrt{1-r^{2}}}-\phi(r, \theta)\right) \\
& -9 \pi-r+5 r \sin \theta-r \cos \theta .
\end{aligned}
$$

For $\varepsilon$ small enough, from Theorem 9 and since $F_{1}$ has only one simple zero at $\rho=3 / 5\left(F_{1}(3 / 5)=0, F_{1}^{\prime}(3 / 5)=15 / 16\right)$, system (22) has a hyperbolic periodic orbit that bifurcates from the circle centered at the origin of radius $3 / 5$.

When $F_{1}=0$, from (18) and Theorem 9, we compute

$$
F_{2}\left(\frac{3}{5}\right)=\frac{125}{16}+\frac{75}{8} \log \frac{9}{8}
$$

and

$$
\frac{1}{2 \pi} \int_{-\pi}^{\pi}\left(G_{1}\left(\frac{3}{5}, \theta\right)-G_{1}\left(\frac{3}{5},-\pi\right)\right) d \theta=-\frac{3}{5}+6 \log \frac{2}{3} .
$$

Consequently, we get the expression of $r(\theta ; 3 / 5, \varepsilon)$ given in the statement where

$$
r_{1}(-\pi ; \rho)=-\frac{22}{3}+10 \log \frac{4}{3} \approx-4.456512613 .
$$

Due to the time rescaling from (22) to (17) the period of this periodic orbit can not be obtained directly from Theorem 9 . Hence the period of the periodic orbit $r(\theta ; \rho, \varepsilon)$ is written as

$$
T(\varepsilon ; \rho)=\int_{-\pi}^{\pi} \frac{r(\theta ; \rho, \varepsilon) d \theta}{(1+r(\theta ; \rho, \varepsilon) \cos \theta)\left(r(\theta ; \rho, \varepsilon)+\varepsilon \alpha_{1}(r(\theta ; \rho, \varepsilon), \theta)\right)},
$$

because

$$
\dot{\theta}=\frac{d \theta}{d t}=(1+r \cos \theta)\left(1+\varepsilon \frac{\alpha_{1}(r, \theta)}{r}\right)
$$


After we substitute $r(\theta ; 3 / 5, \varepsilon)$ for the value given in the statement, the period becomes

$$
T(\varepsilon ; 3 / 5)=\int_{-\pi}^{\pi}\left(T_{0}(3 / 5, \theta)+\varepsilon T_{1}(3 / 5, \theta)\right) d \theta+O\left(\varepsilon^{2}\right),
$$

where $T_{0}(3 / 5, \theta)=5 /(5+3 \cos \theta)$ and

$$
\begin{aligned}
T_{1}\left(\frac{3}{5}, \theta\right)=\frac{25}{3(5+3 \cos \theta)^{2}}( & -20 \sin \theta+3+\cos \theta(5-75 \log 2+30 \log 3 \\
& \left.\left.+15 \log (5+3 \cos \theta)+36 \phi\left(\frac{3}{5}, \theta\right)\right)\right) .
\end{aligned}
$$

Hence, the proof ends by using the expressions of the integrals detailed in Appen$\operatorname{dix}$ A.

In Figure 4 we plot the linear and the numerical approximations of $r(-\pi ; 3 / 5, \varepsilon)$. As can be seen, there is a high dependence in $\varepsilon$ of such values. A small variation in $\varepsilon$ gives a high variation between the approximations of $r(-\pi ; 3 / 5, \varepsilon)$.

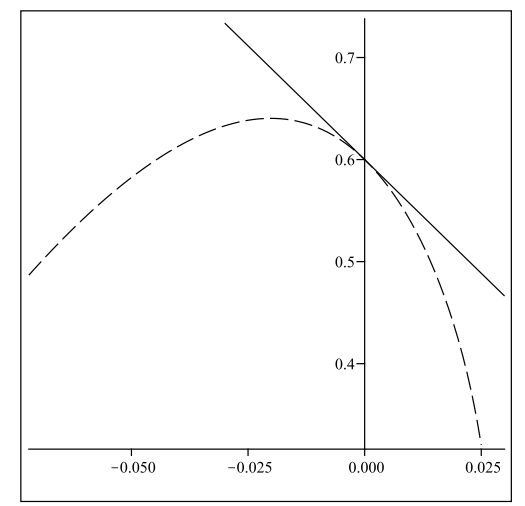

(a)

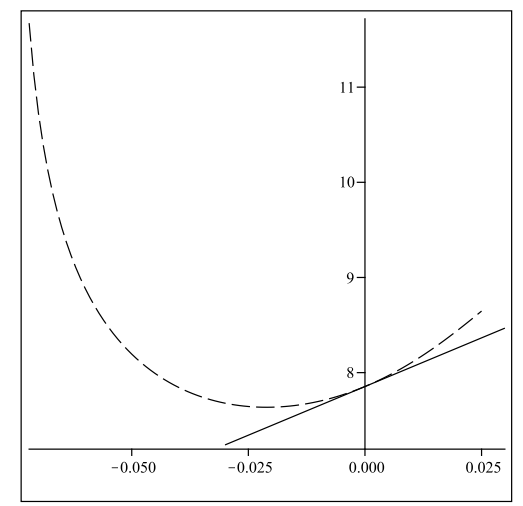

(b)

Figure 4. Comparison between the numerical (doted line) and the linear approximation for $\varepsilon \in[-0.072,0.025]$ for the values of (a) $r(-\pi ; 3 / 5, \varepsilon)$ and (b) $T(\varepsilon ; 3 / 5)$ corresponding to $(22)$.

This example shows the importance of knowing more terms of the series expansion of $r(-\pi ; \rho, \varepsilon)$ in $\varepsilon$. Concretely, it is numerically recommended to get, at least, the term in $\varepsilon^{2}$ particularly when $r_{2}(-\pi)$ is a big number compared with $r_{1}(-\pi)$. This situation causes that numerically, when $\varepsilon$ is not small enough, the approximation of $r(\theta ; \rho, \varepsilon)$ to the real solution is not satisfactory, especially for values of $\rho$ and $r(\theta ; \rho, \varepsilon)$ close to those satisfying $\frac{\partial r}{\partial \varepsilon}(-\pi ; \rho, \varepsilon)=0$, for some $\varepsilon$ close to 0 , which is our case.

Finally, we observe that the periodic orbit given in Proposition 12 only exists when $\varepsilon \in\left(\varepsilon_{H}, \varepsilon_{L}\right)$, where $\varepsilon_{H}=(-23+3 \sqrt{105}) / 260 \approx 0.029773$ and $\varepsilon_{L} \approx$ -0.075011 . More concretely, system (22) has a Hopf bifurcation at the origin when $\varepsilon=\varepsilon_{H}$, while it has a homoclinic connection when $\varepsilon=\varepsilon_{L}$.

3.2. Isochronous quadratic systems. In this section we perturb systems $S_{1}, S_{2}$ and $S_{3}$ given in (12). For these families, there are birational changes of variables, 
$(u, v)=\varphi(x, y)$, so that system (11) is written as

$$
\left\{\begin{aligned}
\dot{u} & =-v+\varepsilon U_{1}(u, v)+\varepsilon^{2} U_{2}(u, v) \\
\dot{v} & =u+\varepsilon V_{1}(u, v,)+\varepsilon^{2} V_{2}(u, v)
\end{aligned}\right.
$$

where $U_{i}, V_{i}$ are rational functions.

Changing to the usual polar coordinates, the above system is written as (17) where $\alpha_{i}$ and $\beta_{i}$ are trigonometric rational functions in $(r, \cos \theta, \sin \theta)$. We consider this equation when $\theta \in[-\pi, \pi]$.

The proof of Theorem 5 follows directly from next result where the number of zeros of the corresponding first and second Poincaré-Pontryagin-Melnikov functions for the three families is given. The first order study coincides with those obtained in $[6,24]$ and we note that no more than two zeros can be found up to the second order of perturbation.

Proposition 13. Let $F_{1}$ and $F_{2}$ be the first and second Poincaré-PontryaginMelnikov functions associated with the quadratic perturbation of each differential equation in (12). The maximum number of zeros of $F_{1}$, taking into account their multiplicity, is $N_{1}(n)$ and of $F_{2}$ (when $\left.F_{1}=0\right)$ is $N_{2}(n)$. They verify that

$\left(S_{1}\right) N_{1}(0)=N_{2}(0)=0, N_{1}(1)=N_{1}(2)=N_{2}(1)=1$ and $N_{2}(2)=2$;

$\left(S_{2}\right) N_{1}(0)=N_{1}(1)=N_{2}(0)=N_{2}(1)=0$ and $N_{1}(2)=N_{2}(2)=2$;

$\left(S_{3}\right) N_{1}(0)=N_{2}(0)=0, N_{1}(1)=N_{2}(1)=1$ and $N_{1}(2)=N_{2}(2)=2$.

Moreover, all these upper bounds are sharp and the functions $F_{1}$ and $F_{2}$ are explicitly obtained.

Proof. The proof is obtained by a case by case study. That is, taking $\left(P_{0}, Q_{0}\right)$ in (11) as the corresponding one in each family of (12) and considering perturbations of degrees $n=0,1,2$.

$\left(S_{1}\right)$ With the change of variables

$$
(x, y)=\left(\frac{2\left(u+u^{2}+v^{2}\right)}{(1+u)^{2}+v^{2}}, \frac{-2 v}{(1+u)^{2}+v^{2}}\right)
$$

and a reversion of time, system (11) writes as (23) where

$$
\begin{aligned}
& U_{i}(u, v)=-\frac{(1+u)^{2}-v^{2}}{2} P_{i}(x(u, v), y(u, v))-v(1+u) Q_{i}(x(u, v), y(u, v)), \\
& V_{i}(u, v)=v(1+u) P_{i}(x(u, v), y(u, v))-\frac{(1+u)^{2}-v^{2}}{2} Q_{i}(x(u, v), y(u, v)),
\end{aligned}
$$

for $i=1,2$. Hence, after changing to polar coordinates, the functions $\alpha_{i}(r, \theta)$ and $\beta_{i}(r, \theta)$ of equation (17) are rational trigonometric functions with denominators power of the expression $1+r^{2}+2 r \cos \theta$. Finally, the change of variables $R=$ $2 r /\left(1+r^{2}\right)$ in (17), allows us to use the integrals of Appendix A to obtain the expressions, only in terms of elementary functions, for the functions $F_{1}(R)$ and $F_{2}(R)$ in (18) and then we apply Theorem 9. The computations are simplified with the auxiliary variable $s=\sqrt{1-R^{2}}$. In this way, we write $\widehat{F}_{i}(s)$ instead of $F_{i}(R)$ and we restrict the study to the interval $(0,1)$.

When $n=2$ and the perturbation polynomials write as

$$
P_{i}(x, y)=\sum_{0 \leq j+k \leq 2} p_{j, k}^{(i)} x^{j} y^{k}, \quad Q_{i}(x, y)=\sum_{0 \leq j+k \leq 2} q_{j, k}^{(i)} x^{j} y^{k}
$$


for $i=1,2$, we have that

$$
\begin{aligned}
& \widehat{F}_{1}(s)=\left(a_{1,2}+b_{1,2} s\right)(1-s) s, \\
& \widehat{F}_{2}(s)=s\left(a_{2,2}(1-s)+b_{2,2}\left(s-s^{2}\right)+c_{2,2} s^{2} \log \frac{2 s}{1+s}\right),
\end{aligned}
$$

where

$$
\begin{aligned}
a_{1,2}= & -p_{0,0}^{(1)}-p_{0,2}^{(1)}-p_{1,0}^{(1)}-p_{2,0}^{(1)}, \\
b_{1,2}= & -p_{0,0}^{(1)}+p_{0,2}^{(1)}+p_{2,0}^{(1)}-q_{0,1}^{(1)}, \\
a_{2,2}= & p_{0,1}^{(1)}\left(-2 p_{0,2}^{(1)}+q_{0,1}^{(1)}\right)-2 p_{0,2}^{(1)}\left(q_{0,0}^{(1)}+q_{0,2}^{(1)}+q_{1,0}^{(1)}+q_{2,0}^{(1)}\right)+p_{1,1}^{(1)} q_{0,1}^{(1)} \\
& -p_{0,0}^{(2)}-p_{0,2}^{(2)}-p_{1,0}^{(2)}-p_{2,0}^{(2)} \\
b_{2,2}= & p_{0,1}^{(1)}\left(2 p_{0,2}^{(1)}-2 p_{2,0}^{(1)}+q_{0,1}^{(1)}+q_{1,1}^{(1)}\right)+p_{0,2}^{(1)}\left(-p_{1,1}^{(1)}+4 q_{0,2}^{(1)}+4 q_{1,0}^{(1)}+6 q_{2,0}^{(1)}\right) \\
& -p_{2,0}^{(1)}\left(p_{1,1}^{(1)}+2 q_{0,2}^{(1)}\right)+q_{1,1}^{(1)}\left(q_{0,0}^{(1)}+2 q_{0,2}^{(1)}+q_{1,0}^{(1)}+2 q_{2,0}^{(1)}\right)+2 q_{0,1}^{(1)} q_{0,2}^{(1)} \\
& -p_{0,0}^{(2)}+p_{0,2}^{(2)}+p_{2,0}^{(2)}-q_{0,1}^{(2)}, \\
c_{2,2}= & 2\left(2 p_{0,2}^{(1)}+q_{1,1}^{(1)}\right)\left(p_{0,1}^{(1)}+2 q_{0,2}^{(1)}+q_{1,0}^{(1)}+2 q_{2,0}^{(1)}\right) .
\end{aligned}
$$

Clearly, we can assume that $a_{i, j}, b_{i, j}$ and $c_{i, j}$ in (26) are arbitrary constants. Similarly, the functions $\widehat{F}_{1}(s)=\widehat{F}_{2}(s)=a_{i, 0}\left(s-s^{3}\right)$ and $\widehat{F}_{1}(s)=\widehat{F}_{2}(s)=$ $\left(a_{i, 1}+b_{i, 1} s\right)\left(s-s^{2}\right)$ when the degree of the perturbations are $n=0,1$, respectively.

The proof of this case finishes using that the set of functions $\{1-s, s-$ $\left.s^{2}, s^{2} \log (2 s /(1+s))\right\}$ forms an Extended Complete Chebyshev system. That is, $\widehat{F}_{2}$, and also $F_{2}$, has at most two zeros and there are examples reaching this bound. Hence, the number of zeros of $(26)$, in $(0,1)$, follows. The other cases in the statement follow straightaway since $\widehat{F}_{i}$ are polynomials. See [22] for more details on ECT-systems.

$\left(S_{2}\right)$ With the change of variables

$$
(x, y)=\left(-\frac{u}{1+u}, \frac{v}{1+u}\right)
$$

and a reversion of time, system (11) is written as (23), where

$$
\begin{aligned}
U_{i}(u, v) & =(1+u)^{2} P_{i}\left(-\frac{u}{1+u}, \frac{v}{1+u}\right), \\
V_{i}(u, v) & =(1+u)\left(v P_{i}\left(-\frac{u}{1+u}, \frac{v}{1+u}\right)-Q_{i}\left(-\frac{u}{1+u}, \frac{v}{1+u}\right)\right),
\end{aligned}
$$

for $i=1,2$. Hence, after we change to polar coordinates, the functions $\alpha_{i}(r, \theta)$ and $\beta_{i}(r, \theta)$ of equation (17) are rational trigonometric functions with powers of the expression $1+r \cos \theta$ as a denominator.

The functions $F_{1}(r)$ and $F_{2}(r)$ given in (18) are computed from Theorem 9 using the integrals of Appendix A. As in the previous case we use the auxiliary variable $s=\sqrt{1-r^{2}}$ and we write $\widehat{F}_{i}(s)$ instead of $F_{i}(r)$ restricting our attention to the interval $(0,1)$. Hence, the functions $\widehat{F}_{i}, i=1,2$, are $a_{i, 0}\left(1-s^{2}\right), a_{i, 1}\left(1-s^{2}\right)$ and $\left(a_{i, 2}+b_{i, 2} s+c_{i, 2} s^{2}\right)(1-s)$ when $n=0,1,2$, respectively. Here, $a_{i, j}, b_{i, j}$ and $c_{i, j}$ can be taken as arbitrary constants. Hence, for this case the statement immediately follows since $\widehat{F}_{i}$ are polynomials. 
$\left(S_{3}\right)$ This case follows in the same way as the previous cases taking the change of variables

$$
(x, y)=\left(\frac{3 v}{8(1+u)}, \frac{3\left(2 u+2 u^{2}+v^{2}\right)}{16(1+u)^{2}}\right),
$$

and system (11) is written as (23) where

$$
\begin{aligned}
& U_{i}(u, v)=-\frac{8}{3}(1+u)\left(v P_{i}(x(u, v), y(u, v))-(1+u) Q_{i}(x(u, v), y(u, v))\right), \\
& V_{i}(u, v)=\frac{8}{3}\left(\left(1+u-v^{2}\right) P_{i}(x(u, v), y(u, v))+v Q_{i}(x(u, v), y(u, v))\right),
\end{aligned}
$$

for $i=1,2$. The functions $\widehat{F}_{i}, i=1,2$, are $a_{i, 0}\left(1-s^{2}\right),\left(a_{i, 1}(1+s)+c_{i, 1} s^{2}\right)(1-s)$ and $\left(a_{i, 2}+b_{i, 2} s+c_{i, 2} s^{2}\right)(1-s)$ when $n=0,1,2$, respectively. Furthermore, they are linear combination of 1,2 and 3 functions, respectively. Arguing, as in the family $S_{1}$, on ECT-systems for each $\widehat{F}_{i}$, we conclude that they have $0,1,2$ zeros respectively.

The study of first and second order of the last result provides at most two hyperbolic periodic orbits bifurcating from the isochronous centers given in (12). Next result shows quadratic perturbations realizing them when the first PoincaréPontryagin-Melnikov vanishes identically.

Proposition 14. The following perturbed isochronous systems have two hyperbolic periodic orbits.

$$
\begin{aligned}
& \begin{array}{l}
S_{1}^{\varepsilon}\left\{\begin{aligned}
\dot{x}= & -y+x y+\varepsilon\left(1-2 x+8 y-12 x^{2}\right. \\
& \left.+8(76+150 a-27 b) x y+13 y^{2}\right)+\varepsilon^{2}(-208-216 a \\
\dot{y}= & x-\frac{1}{2} x^{2}+\frac{1}{2} y^{2},
\end{aligned}\right. \\
\text { where } a=\log (3 / 4) \text { and } b=\log (3 / 5) . \\
S_{2}^{\varepsilon} \begin{cases}\dot{x}= & -y(1+x)+\varepsilon\left(\frac{2}{3}+2 x-\frac{3}{2} x y+x^{2}\right)+\frac{13}{2} \varepsilon^{2} y^{2}, \\
\dot{y}= & x-y^{2}+\varepsilon\left(3+x y+3 x^{2}\right) .\end{cases}
\end{array} \\
& S_{3}^{\varepsilon}\left\{\begin{array}{l}
\dot{x}=y+\frac{4}{3} x^{2}+\varepsilon\left(-41 x-x^{2}+\frac{908}{9} x y+\frac{140}{9} y^{2}\right), \\
\dot{y}=-x\left(1-\frac{16}{3} y\right)+\varepsilon\left(1+33 y-2 x^{2}-\frac{1816}{9} y^{2}\right) .
\end{array}\right.
\end{aligned}
$$

Proof. The same steps as in Proposition 13 provide $\widehat{F}_{1}=0$ for the three families. Furthermore, the second Poincaré-Pontryagin-Melnikov functions are

$$
\widehat{F}_{2}(s)=54(8 a-3 b)(1-s) s-6(328 a-45 b)(1-s) s^{2}+832 s^{3} \log \frac{2 s}{1+s}
$$

for $S_{1}^{\varepsilon}$ and

$$
\widehat{F}_{2}(s)=(3 s-1)(2 s-1)(s-1)
$$

for $S_{2}^{\varepsilon}$ and $S_{3}^{\varepsilon}$. Then, the roots of the corresponding $\widehat{F}_{2}$ are $s_{1}=3 / 5, s_{2}=9 / 41$ and $s_{1}=1 / 2, s_{2}=1 / 3$, respectively. The proof follows from Theorem 9 since they are simple roots.

Finally we present three concrete examples where hyperbolic periodic orbits bifurcate from the isochronous quadratic families considered in this paper. Moreover the periods of such solutions are also done. 
Proposition $15\left(S_{1}\right)$. The perturbed isochronous system

$$
\left\{\begin{array}{l}
\dot{x}=-y+x y+2 \varepsilon\left(-3 x-117 y+15 x^{2}+4 x y\right) \\
\dot{y}=x-\frac{1}{2} x^{2}+\frac{1}{2} y^{2}
\end{array}\right.
$$

has a hyperbolic periodic orbit with period $T(\varepsilon)=2 \pi-104 \pi \varepsilon+O\left(\varepsilon^{2}\right)$ and is written as

$$
\begin{aligned}
& x(\theta ; \varepsilon)=\frac{1+3 \cos \theta}{5+3 \cos \theta}+\varepsilon \frac{(3+5 \cos \theta)}{(5+3 \cos \theta)^{2}}\left(\frac{192 \Psi_{0}(\theta)}{5}+\frac{3 \Psi_{1}(\theta)}{(5+3 \cos \theta)}\right)+O\left(\varepsilon^{2}\right), \\
& y(\theta ; \varepsilon)=\frac{-3 \sin \theta}{5+3 \cos \theta}-\varepsilon \frac{4 \sin \theta}{(5+3 \cos \theta)^{2}}\left(\frac{192 \Psi_{0}(\theta)}{5}+\frac{3 \Psi_{1}(\theta)}{(5+3 \cos \theta)}\right)+O\left(\varepsilon^{2}\right) .
\end{aligned}
$$

where

$$
\begin{aligned}
& \Psi_{0}(\theta)=3 \phi(3 / 5, \theta)-15 \lambda(3 / 5, \theta)+5 \log (72 / 125), \\
& \Psi_{1}(\theta)=64+963 \cos \theta-180 \sin \theta+565 \cos ^{2} \theta-60 \cos \theta \sin \theta .
\end{aligned}
$$

The functions $\phi$ and $\lambda$ are defined in (5).

Proof. We apply the procedure of the proof of Proposition 13, for the family $S_{1}$, to system (31) which moves to (23). Hence, we get

$$
F_{1}(R)=-3\left(9-5 R^{2}-9 \sqrt{1-R^{2}}\right) \sqrt{1-R^{2}},
$$

which has a simple zero at $\rho=3 / 5\left(F_{1}^{\prime}(3 / 5)=-9 / 5\right)$. Theorem 9 guarantees that system (31) has a hyperbolic periodic orbit, for $\varepsilon$ small enough, bifurcating from $\rho=3 / 5$. Moreover, $R_{1}(-\pi ; 3 / 5)=(-2004+1536 \log 3) / 25$ and

$$
R\left(\theta ; \frac{3}{5}, \varepsilon\right)=\frac{3}{5}+\varepsilon \frac{768}{125}\left(\Psi_{0}(\theta)+\frac{5 \Psi_{1}(\theta)}{64(5+3 \cos \theta)}\right)+O\left(\varepsilon^{2}\right),
$$

where $\Psi_{i}$ are defined in the statement. Consequently

$$
r\left(\theta ; \frac{1}{3}, \varepsilon\right)=\frac{1}{3}+\varepsilon \frac{64}{15}\left(\Psi_{0}(\theta)+\frac{5 \Psi_{1}(\theta)}{64(5+3 \cos \theta)}\right)+O\left(\varepsilon^{2}\right),
$$

and the period is written as in the statement.

The proof finishes by substituting, in the inverse of the change of variables (24), the values $u=r(\theta ; 1 / 3, \varepsilon) \cos \theta, v=r(\theta ; 1 / 3, \varepsilon) \sin \theta$ and by expanding in power series of $\varepsilon$.

Proposition $16\left(S_{2}\right)$. The perturbed isochronous system

$$
\left\{\begin{array}{l}
\dot{x}=-y(1+x)+\varepsilon\left(\frac{1}{10} x+y-\frac{262}{5} x^{2}+\frac{41}{2} y^{2}\right), \\
\dot{y}=x-y^{2}+6 \varepsilon x^{2}
\end{array}\right.
$$

has two hyperbolic periodic orbits that parametrize, for $\theta \in[-\pi, \pi]$, as

$$
x\left(\theta ; \rho_{i}\right)=-\frac{r\left(\theta ; \rho_{i}, \varepsilon\right) \cos \theta}{1+r\left(\theta ; \rho_{i}, \varepsilon\right) \cos \theta}, \quad y\left(\theta ; \rho_{i}\right)=\frac{r\left(\theta ; \rho_{i}, \varepsilon\right) \sin \theta}{1+r\left(\theta ; \rho_{i}, \varepsilon\right) \cos \theta}
$$


for $i=1,2$ and $\rho_{1}=3 / 5$ and $\rho_{2}=9 / 41$. Here

$$
\begin{aligned}
r\left(\theta ; \rho_{1}, \varepsilon\right)=\rho_{1}+\varepsilon & \left(-\frac{169997}{540}-\frac{22910}{3} \log 2+\frac{45700}{9} \log 3+10 \log 5\right. \\
& -\frac{159}{25} \cos \theta+\frac{2331}{50} \sin \theta+\frac{3}{4} \cos (2 \theta)-\frac{219}{20} \sin (2 \theta) \\
& \left.-\frac{8736}{125} \phi\left(\rho_{1}, \theta\right)+10 \log \left(1+\rho_{1} \cos \theta\right)\right)+O\left(\varepsilon^{2}\right), \\
r\left(\theta ; \rho_{2}, \varepsilon\right)=\rho_{2}+\varepsilon & \left(\frac{82}{3} \log 2-\frac{328}{3} \log 3+\frac{82}{3} \log 41\right. \\
& -\frac{10167}{1681} \cos \theta+\frac{1166319}{16810} \sin \theta+\frac{45}{164} \cos (2 \theta)-\frac{657}{164} \sin (2 \theta) \\
& \left.-\frac{524160}{1681} \phi\left(\rho_{2}, \theta\right)+\frac{82}{3} \log \left(1+\rho_{2} \cos \theta\right)\right)+O\left(\varepsilon^{2}\right) .
\end{aligned}
$$

Here the function $\phi$ is defined in (5). Furthermore, their periods are written as

$$
T\left(\varepsilon ; \rho_{1}\right)=2 \pi-\frac{4}{3} \pi \varepsilon+O\left(\varepsilon^{2}\right) \quad \text { and } \quad T\left(\varepsilon ; \rho_{2}\right)=2 \pi+\frac{209}{270} \pi \varepsilon+O\left(\varepsilon^{2}\right) .
$$

Proof. Following the proof of Proposition 13 for the family $S_{2}$ we obtain the first Poincaré-Pontryagin-Melnikov function

$$
F_{1}(r)=\frac{729-934 r^{2}+205 r^{4}-\left(729-569 r^{2}\right) \sqrt{1-r^{2}}}{10 \sqrt{1-r^{2}}} .
$$

It is easy to check that $F_{1}$ has two positive zeros, $\rho_{1}=3 / 5$ and $\rho_{2}=9 / 41$, which are simple $\left(F_{1}^{\prime}\left(\rho_{1}\right)=-27 / 50, F_{1}^{\prime}\left(\rho_{2}\right)=81 / 4100\right)$. Hence, from Theorem 9 , system (32) has two hyperbolic periodic orbits bifurcating from (33) for $\varepsilon=0$ for those values of $\rho_{i}$. Consequently the expression of $r\left(\theta ; \rho_{i}, \varepsilon\right)$ given in the statement is obtained. In particular

$$
\begin{aligned}
& r_{1}\left(-\pi ; \rho_{1}\right)=-\frac{207697}{675}-\frac{22880}{3} \log 2+\frac{45700}{9} \log 3, \\
& r_{1}\left(-\pi ; \rho_{2}\right)=\frac{42513}{6724}+164 \log 2-\frac{328}{3} \log 3 .
\end{aligned}
$$

The proof finishes by using the change of coordinates (28) and computing the periods also from Theorem 9 and Appendix A.

Proposition $17\left(S_{3}\right)$. System

$$
\left\{\begin{array}{l}
\dot{x}=y+\frac{4}{3} x^{2}+\varepsilon\left(3+x^{2}+\frac{3652}{9} x y\right) \\
\dot{y}=\frac{-x(3-16 y)}{3}+\varepsilon\left(\frac{1}{100}+6 x^{2}+\frac{18616}{225} y^{2}\right),
\end{array}\right.
$$

has two hyperbolic periodic orbits bifurcating from the curves that parametrize, for $\theta \in[-\pi, \pi]$, as

$$
x\left(\theta ; \rho_{i}\right)=\frac{3 \rho_{i} \sin \theta}{8\left(1+\rho_{i} \cos \theta\right)}, \quad y\left(\theta ; \rho_{i}\right)=\frac{12 \rho_{i}\left(2 \cos \theta+\rho_{i}\left(1+\cos ^{2} \theta\right)\right)}{64\left(1+\rho_{i} \cos \theta\right)^{2}}
$$

for $i=1,2$ and $\rho_{1}=3 / 5$ and $\rho_{2}=9 / 41$. Their periods are written as

$$
T\left(\varepsilon ; \rho_{1}\right)=2 \pi-\pi \frac{193}{12} \varepsilon+O\left(\varepsilon^{2}\right) \quad \text { and } \quad T\left(\varepsilon ; \rho_{2}\right)=2 \pi-\pi \frac{1729}{108} \varepsilon+O\left(\varepsilon^{2}\right) .
$$


Moreover, they pass through the points $\left(0, y_{ \pm}\left(\varepsilon, \rho_{i}\right)\right)$ where

$$
\begin{gathered}
y_{-}\left(\varepsilon ; \rho_{1}\right)=-\frac{9}{16}+\varepsilon\left(-\frac{25906039}{18432}+\frac{1235}{2} \log 2-\frac{4955}{12} \log 3\right)+O\left(\varepsilon^{2}\right), \\
y_{+}\left(\varepsilon ; \rho_{1}\right)=\frac{9}{64}+\varepsilon\left(\frac{25505143}{294912}-\frac{2485}{64} \log 2+\frac{4955}{192} \log 3\right)+O\left(\varepsilon^{2}\right), \\
y_{-}\left(\varepsilon ; \rho_{2}\right)=-\frac{27}{256}+\varepsilon\left(\frac{11016651275}{7077888}-\frac{13793425}{2304} \log 2+\frac{6901325}{1152} \log 3\right. \\
\left.\quad-\frac{3455275}{2304} \log 5\right)+O\left(\varepsilon^{2}\right), \\
y_{+}\left(\varepsilon ; \rho_{2}\right)=\frac{27}{400}+\varepsilon\left(-\frac{443071427}{691200}+\frac{22099}{9} \log 2-\frac{552106}{225} \log 3\right. \\
\left.+\frac{137842}{225} \log 5\right)+O\left(\varepsilon^{2}\right) .
\end{gathered}
$$

Proof. The first steps follow as in the proof of Proposition 13 for the family $S_{3}$. System (34) transforms, via the rational change of coordinates (29), to a linear center with the rational perturbation (30). Moving to polar coordinates we can proceed analogously and obtain

$$
F_{1}(r)=\frac{2\left(729-934 r^{2}+205 r^{4}-\left(729-569 r^{2}\right) \sqrt{1-r^{2}}\right)}{25 \sqrt{1-r^{2}}} .
$$

It can be checked that $F_{1}$ has only two positive zeros, $\rho_{1}=3 / 5$ and $\rho_{2}=9 / 41$, which are simple $\left(F_{1}^{\prime}\left(\rho_{1}\right)=54 / 125, F_{1}^{\prime}\left(\rho_{2}\right)=-81 / 5125\right)$. Hence, from Theorem 9 , system (34) has two hyperbolic periodic orbits bifurcating from (35) for these values of $\rho_{i}$. Consequently the expression of $r\left(\theta ; \rho_{i}, \varepsilon\right)$ is obtained up to first order, where

$$
\begin{aligned}
& r_{1}\left(-\pi ; \rho_{1}\right)=\frac{25906039}{43200}-\frac{3952}{15} \log 2+\frac{7928}{45} \log 3, \\
& r_{1}\left(-\pi ; \rho_{2}\right)=-\frac{11016651275}{4357152}+\frac{10765600}{1107} \log 2-\frac{10772800}{1107} \log 3+\frac{2696800}{1107} \log 5 .
\end{aligned}
$$

Evaluating the radius at $\theta=-\pi$ and $\theta=0$, we obtain the intersection points, in $(u, v)$-coordinates, with the $u$-axis. The expressions of the values of $\left(0, y_{ \pm}(\varepsilon)\right)$ given in the statement, using the change of coordinates (29), follow. The proof finishes computing the periods also from Theorem 9 .

\section{Simultaneity}

Next result proves the second part of the statement of Theorem 5. That is the realizable configurations of simultaneous bifurcation of limit cycles for family $S_{1}$, see (12), up to first and second order. In fact, it revisits the first order studies done in $[6,23]$. A first order perturbation but up to degree $n$ is considered in [11]. Other simultaneity studies up to first order for quadratic systems are [5, 8, 25].

Proposition 18. Let $P_{i}, Q_{i}, i=1,2$ be quadratic polynomials. The realizable configuration of limit cycles that bifurcate from the level curves of the center, when 
$\varepsilon=0$, for system

$$
\left\{\begin{array}{l}
\dot{x}=-y+x y+\varepsilon P_{1}(x, y)+\varepsilon^{2} P_{2}(x, y) \\
\dot{y}=x-\frac{1}{2} x^{2}+\frac{1}{2} y^{2}+\varepsilon Q_{1}(x, y)+\varepsilon^{2} Q_{2}(x, y),
\end{array}\right.
$$

are $(1,0),(0,1)$ and $(1,1)$ up to first order and $(2,0),(1,1)$ and $(0,2)$ up to second order.

Proof. System (36) has two isochronous centers at $(0,0)$ and $(2,0)$ that are, in some sense, symmetric. In fact, the change of variables $(z, w)=(-x+2, y)$ moves system (36), with $P_{i}$ and $Q_{i}$ defined in (25), to

$$
\left\{\begin{array}{l}
\dot{z}=-w+z w+\varepsilon \bar{P}_{1}(z, w)+\varepsilon^{2} \bar{P}_{2}(z, w) \\
\dot{w}=z-\frac{1}{2} z^{2}+\frac{1}{2} w^{2}+\varepsilon \bar{Q}_{1}(z, w)+\varepsilon^{2} \bar{Q}_{2}(z, w)
\end{array}\right.
$$

with $\bar{P}_{i}(z, w)=\sum_{0 \leq j+k \leq 2} \bar{p}_{j, k}^{(i)} z^{j} w^{k}, \bar{Q}_{i}(z, w)=\sum_{0 \leq j+k \leq 2} \bar{q}_{j, k}^{(i)} z^{j} w^{k}$ where

$$
\begin{array}{lll}
\bar{p}_{0,0}^{(i)}=-p_{0,0}^{(i)}-2 p_{1,0}^{(i)}-4 p_{2,0}^{(i)}, & \bar{p}_{1,0}^{(i)}=p_{1,0}^{(i)}+4 p_{2,0}^{(i)}, & \bar{p}_{0,1}^{(i)}=-p_{0,1}^{(i)}-2 p_{1,1}^{(i)}, \\
\bar{q}_{0,0}^{(i)}=q_{0,0}^{(i)}+2 q_{1,0}^{(i)}+4 q_{2,0}^{(i)}, & \bar{q}_{1,0}^{(i)}=-4 q_{2,0}^{(i)}-q_{1,0}^{(i)}, & \bar{q}_{0,1}^{(i)}=2 q_{1,1}^{(i)}+q_{0,1}^{(i)}, \\
\bar{p}_{2,0}^{(i)}=-p_{2,0}^{(i)}, & \bar{p}_{1,1}^{(i)}=p_{1,1}^{(i)}, & \bar{p}_{0,2}^{(i)}=-p_{0,2}^{(i)}, \\
\bar{q}_{2,0}^{(i)}=q_{2,0}^{(i)}, & \bar{q}_{1,1}^{(i)}=-q_{1,1}^{(i)}, & \bar{q}_{0,2}^{(i)}=q_{0,2}^{(i)},
\end{array}
$$

for $i=1,2$.

The first and second Poincaré-Pontryagin-Melnikov functions for the center located at the origin of (36) are given in the proof of Proposition 13. Consequently, we can also obtain them for the center located at $(2,0)$ of $(36)$ (i.e. the origin of (37)) using the symmetry and changing $p_{j, k}^{(i)}$ and $q_{j, k}^{(i)}$ by $\bar{p}_{j, k}^{(i)}$ and $\bar{q}_{j, k}^{(i)}$, respectively, in expressions (26) and (27). Let us call, following the notation of the proof of Proposition $13, F_{11}$ and $F_{21}$ (respectively $F_{12}$ and $F_{22}$ ) to the functions $\widehat{F}_{1}$ and $\widehat{F}_{2}$, given in (26), for system (36) (respectively (37)).

In a simplified way we may write

$$
F_{11}(s)=\left(a_{0}+a_{1} s\right)(1-s) s, \quad F_{12}(s)=\left(-a_{0}+b_{1} s\right)(1-s) s,
$$

for some values of $a_{0}, a_{1}$ and $b_{1}$ that are independent linear combinations of the coefficients of $P_{1}$ and $Q_{1}$. We remark that although $F_{11}$ and $F_{12}$ have four relevant coefficients only three of them are really independent. But this does not prevent to prove that the realizable configurations are only $(1,0),(0,1)$ and $(1,1)$ up to first order study.

The second order study needs two cases, when only one or both of $\widehat{F}_{1}$ vanish. In the first case, assuming that only $F_{11}=0$ (i.e. $a_{0}=a_{1}=0$ and $b_{1} \neq 0$ ), we have that $F_{21}$, see (26), can have at most two zeros and $F_{12}$ does not vanish. Therefore the realizable configurations are only $(2,0)$ and, by symmetry, $(0,2)$. Finally, it can be seen that, in the second case, the conditions $a_{0}=a_{1}=b_{1}=0$ imply that the coefficient of the term with log, in (26), vanishes for both $F_{21}$ and $F_{22}$. Hence the realizable configurations are the same that the ones obtained with a first order perturbation. 


\section{Final COMments}

Concerning the families in this paper we present the main obstacles for the study of their higher order perturbation in $\varepsilon$.

As it can be seen in [32], where polynomial perturbations of a linear center are considered, the Poincaré-Pontryagin-Melnikov functions of higher order can be obtained without additionally difficulties. Such a generating functions can be obtained because the involved integrals only depend polynomially on $r, \sin \theta$ and $\cos \theta$. This is in the polynomial case where the iterated integrals, in the sense of Chen-Gavrilov, of algebraically relative exact polynomial forms, reduce to an integral of a polynomial 1-form. See [14].

Regarding the families studied in this work, we have considered rational perturbations up to a second order in $\varepsilon$ and, as we have shown, the computation of the Poincaré-Pontryagin-Melnikov functions, i.e. the computation of some integrals is the main obstacle. The primitives appear depending on the functions $\lambda$ and $\phi$ as they are defined in (5). As we mentioned before, to go further in the order of perturbation we need to get the expressions, in particular, of the integrals (4). In fact, this is the main obstruction because our procedure uses explicit expressions of all of them.

For instance, to get the approximation of second order it is necessary to obtain the expression of $F_{3}$. This function cannot be obtained, in general, in polar coordinates because it depends on $\int_{-\pi}^{\pi} r_{2}(\psi) d \psi$ and $\int_{-\pi}^{\pi} r_{1}^{2}(\psi) d \psi$, for example. We point out that forcing primitives like

$$
\int_{-\pi}^{\theta} \frac{\cos (k \psi) \lambda(r, \psi)}{(1+r \cos \psi)^{\ell}} d \psi
$$

not to appear, i.e. by an appropriate choice of the coefficients of the perturbation, the function $F_{3}$ can be computed. For this particular selection, we obtain only two zeros for the quadratic perturbations and five for the cubic, one more than those obtained for $F_{2}$ in Proposition 3.

\section{Appendix A. Computations of the integrals}

This appendix is devoted to providing the expressions and properties for the functions required to get the results of the previous sections. They appear in the first and second order studies, using the Poincaré-Pontryagin-Melnikov theory, for all the perturbation problems described in this paper.

For each pair of natural numbers $k$ and $\ell$, we define the following functions:

$$
\begin{aligned}
\mathcal{S}_{k, \ell}(r, \theta) & =\int_{-\pi}^{\theta} \frac{\sin (k \psi)}{(1+r \cos \psi)^{\ell}} d \psi, & \mathcal{C}_{k, \ell}(r, \theta) & =\int_{-\pi}^{\theta} \frac{\cos (k \psi)}{(1+r \cos \psi)^{\ell}} d \psi, \\
s_{k, \ell}(r) & =\frac{1}{2 \pi} \int_{-\pi}^{\pi} \frac{\sin (k \psi)}{(1+r \cos \psi)^{\ell}} d \psi, & c_{k, \ell}(r) & =\frac{1}{2 \pi} \int_{-\pi}^{\pi} \frac{\cos (k \psi)}{(1+r \cos \psi)^{\ell}} d \psi, \\
s_{k, \ell}^{\phi}(r) & =\frac{1}{2 \pi} \int_{-\pi}^{\pi} \frac{\sin (k \psi) \phi(r, \psi)}{(1+r \cos \psi)^{\ell}} d \psi, & c_{k, \ell}^{\phi}(r) & =\frac{1}{2 \pi} \int_{-\pi}^{\pi} \frac{\cos (k \psi) \phi(r, \psi)}{(1+r \cos \psi)^{\ell}} d \psi, \\
s_{k, \ell}^{\lambda}(r) & =\frac{1}{2 \pi} \int_{-\pi}^{\pi} \frac{\sin (k \psi) \lambda(r, \psi)}{(1+r \cos \psi)^{\ell}} d \psi, & c_{k, \ell}^{\lambda}(r) & =\frac{1}{2 \pi} \int_{-\pi}^{\pi} \frac{\cos (k \psi) \lambda(r, \psi)}{(1+r \cos \psi)^{\ell}} d \psi .
\end{aligned}
$$

Here $r \in(-1,1), \theta \in[-\pi, \pi]$ and $\phi$ and $\lambda$ are defined in (5).

Not all of these primitives can be obtained in terms of elementary transcendental functions. In this section we give expressions, some are recursive, for them. In 
particular, for concrete values of $k$ and $\ell$, the functions (40) can be expressed in terms of $r, \phi(r, \theta), \lambda(r, \theta), \cos \theta$ and $\sin \theta$. Specifically the definitions for $\phi$ and $\lambda$ are motivated from the primitives $\mathcal{C}_{0,1}$ and $\mathcal{S}_{1,1}$, respectively. We have modified them to satisfy properties on symmetry and periodicity like the trigonometric usual functions. The qualitative behavior of $\phi$ and $\lambda$ can be seen in Figure 5, where we have drawn both for a given value of $r$. Next lemma deal with these properties.
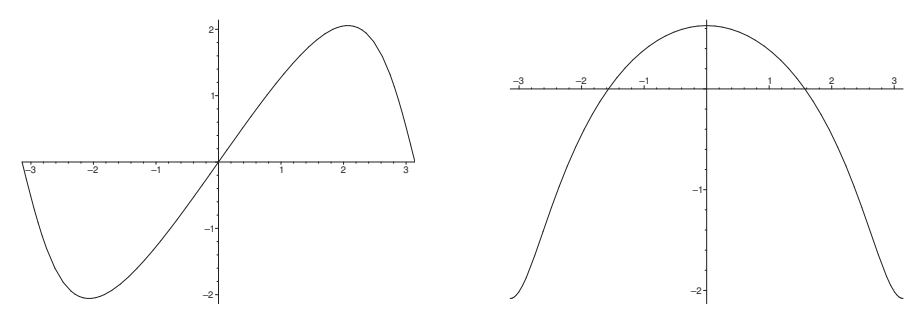

Figure 5. Plot of the functions $\phi$ and $\lambda$ when $r=7 / 8$.

Lemma 19. The functions $\phi$ and $\lambda$, defined in (5), satisfy the following properties:

$$
\begin{aligned}
\phi(r,-\theta) & =-\phi(r, \theta), & \lambda(r,-\theta) & =\lambda(r, \theta), \\
\phi(r, \theta+2 \pi) & =\phi(r, \theta), & \lambda(r, \theta+2 \pi) & =\lambda(r, \theta), \\
\int_{-\pi}^{\pi} \phi(r, \psi) d \psi & =0, & \int_{-\pi}^{\pi} \lambda(r, \psi) d \psi & =2 \pi \log \left(\frac{1+\sqrt{1-r^{2}}}{2}\right) .
\end{aligned}
$$

Moreover

$$
\begin{aligned}
\frac{\partial}{\partial r} \phi(r, \theta) & =\frac{1}{1-r^{2}}\left(r \phi(r, \theta)+\frac{\sin \theta}{1+r \cos \theta}\right) \\
\frac{\partial}{\partial \theta} \phi(r, \theta) & =\frac{1}{\sqrt{1-r^{2}}}-\frac{1}{1+r \cos \theta}
\end{aligned}
$$

Some of the functions defined in (40) vanish due to the symmetry with respect to $\theta$. The following result is a summary of this fact.

Lemma 20. Let $\lambda$ and $\phi$ be the functions defined in (5). For each $k$, $\ell$ and $s$ natural numbers, we get:

$$
\begin{array}{ll}
\int_{-\pi}^{\pi} \frac{\sin (k \psi) \phi(r, \psi)^{2 s}}{(1+r \cos \psi)^{\ell}} d \psi \equiv 0, & \int_{-\pi}^{\pi} \frac{\cos (k \psi) \phi(r, \psi)^{2 s+1}}{(1+r \cos \psi)^{\ell}} d \psi \equiv 0, \\
\int_{-\pi}^{\pi} \frac{\sin (k \psi) \lambda(r, \psi)^{s}}{(1+r \cos \psi)^{\ell}} d \psi \equiv 0, & \text { and } \quad c_{k, \ell}^{\phi}(r) \equiv 0, \quad s_{k, \ell}(r) \equiv 0, \quad s_{k, \ell}^{\lambda}(r) \equiv 0 .
\end{array}
$$

In the following results we give expressions for the functions defined at the beginning of this appendix. 
Proposition 21. The functions $\mathcal{S}_{k, \ell}$ and $\mathcal{C}_{k, \ell}$, defined in (40), are written as

$$
\begin{aligned}
\mathcal{S}_{k, \ell}(r, \theta)= \begin{cases}0 & k=0, \ell \geq 0, \\
-\left(\cos (k \theta)-(-1)^{k}\right) k^{-1} & k \geq 1, \ell=0, \\
-(\log (1+r \cos \theta)-\log (1-r)) r^{-1} & k=1, \ell=1, \\
\left((1+r \cos \theta)^{1-\ell}-(1-r)^{1-\ell}\right)(r(\ell-1))^{-1} & k=1, \ell \geq 2, \\
2\left(\mathcal{S}_{k-1, \ell-1}(r, \theta)-\mathcal{S}_{k-1, \ell}(r, \theta)\right) r^{-1}-\mathcal{S}_{k-2, \ell}(r, \theta) & k \geq 2, \ell \geq 2,\end{cases} \\
\mathcal{C}_{k, \ell}(r, \theta)= \begin{cases}\theta+\pi & k=0, \ell=0, \\
-\phi(r, \theta)+(\theta+\pi)\left(1-r^{2}\right)^{-\frac{1}{2}} & k=0, \ell=1, \\
\mathcal{C}_{0, \ell-1}(r, \theta)+\frac{r}{l-1} \frac{\partial}{\partial r} \mathcal{C}_{0, \ell-1}(r, \theta) & k=0, \ell \geq 2, \\
\sin (k \theta) k^{-1} & k \geq 1, \ell=0, \\
\left(\mathcal{C}_{0, \ell-1}(r, \theta)-\mathcal{C}_{0, \ell}(r, \theta)\right) r^{-1} & k=1, \ell \geq 1, \\
2\left(\mathcal{C}_{k-1, \ell-1}(r, \theta)-\mathcal{C}_{k-1, \ell}(r, \theta)\right) r^{-1}-\mathcal{C}_{k-2, \ell}(r, \theta) & k \geq 2, \ell \geq 2,\end{cases}
\end{aligned}
$$

when $r \neq 0$. Furthermore, $\mathcal{S}_{k, \ell}(0, \theta)=\mathcal{S}_{k, 0}(r, \theta)$ and $\mathcal{C}_{k, \ell}(0, \theta)=\mathcal{C}_{k, 0}(r, \theta)$.

Proof. The expressions of $\mathcal{S}_{k, \ell}(r, \theta)$ for $k=0,1$ are obtained by using direct integration. When $k \geq 2$ and $l \geq 2$, from its definition and by using elementary transformations, we get

$$
\begin{aligned}
\mathcal{S}_{k-1, \ell-1}(r, \theta) & =\int_{-\pi}^{\theta} \frac{\sin ((k-1) \psi)}{(1+r \cos \psi)^{\ell-1}} d \psi=\int_{-\pi}^{\theta} \frac{\sin ((k-1) \psi)(1+r \cos \psi)}{(1+r \cos \psi)^{\ell}} d \psi \\
& =\int_{-\pi}^{\theta} \frac{\sin ((k-1) \psi)}{(1+r \cos \psi)^{\ell}} d \psi+r \int_{-\pi}^{\theta} \frac{\sin ((k-1) \psi) \cos \psi}{(1+r \cos \psi)^{\ell}} d \psi .
\end{aligned}
$$

Using the identity $2 \sin ((k-1) \psi) \cos \psi=\sin (k \psi)+\sin ((k-2) \psi)$ last equality is written as

$$
\mathcal{S}_{k-1, \ell-1}(r, \theta)=\mathcal{S}_{k-1, \ell}(r, \theta)+\frac{1}{2} r \mathcal{S}_{k, \ell}(r, \theta)+\frac{1}{2} r \mathcal{S}_{k-2, \ell}(r, \theta) .
$$

The expression of $\mathcal{S}_{k, \ell}(r, \theta)$, in the statement, comes from solving it from the above relation.

The expression of $\mathcal{C}_{k, 0}(r, \theta)$ comes from direct integration; while the $\mathcal{C}_{0,1}$ is obtained by using the change of variables $\tan (\psi / 2)=\alpha$ and by taking into account the definition of the function $\phi$ given in (5). The expression of $\mathcal{C}_{0, \ell}(r, \theta), \ell \geq 2$ follows deriving with respect to $r$. The proof ends providing similar arguments as those used in the case of $\mathcal{S}_{k, \ell}(r, \theta)$.

Next corollary comes directly by evaluating at $\theta=\pi$ and dividing by $2 \pi$ the expression of $\mathcal{C}_{k, \ell}$ appearing in last proposition.

Corollary 22. The function $c_{k, \ell}$, defined in (40), is expressed as

$$
c_{k, \ell}(r)= \begin{cases}1 & k=0, \ell=0, \\ \left(1-r^{2}\right)^{-\frac{1}{2}} & k=0, \ell=1, \\ c_{0, \ell-1}(r)+\frac{r}{\ell-1} \frac{d}{d r} c_{0, \ell-1}(r) & k=0, \ell \geq 2, \\ 0 & k \geq 1, \ell=0, \\ \left(c_{0, \ell-1}(r)-c_{0, \ell}(r)\right) r^{-1} & k=1, \ell \geq 1, \\ 2\left(c_{k-1, \ell-1}(r)-c_{k-1, \ell}(r)\right) r^{-1}-c_{k-2, \ell}(r) & k \geq 2, \ell \geq 1,\end{cases}
$$


when $r \neq 0$. Also, $c_{0, \ell}(0)=1$ and $c_{k, \ell}(0)=0$.

Proposition 23. The function $c_{k, \ell}^{\lambda}$, defined in (40), is written as

$$
c_{k, \ell}^{\lambda}(r)= \begin{cases}\log \left(1+\sqrt{1-r^{2}}\right)-\log 2 & k=0, \ell=0, \\ \frac{1}{\sqrt{1-r^{2}}} \log \left(\frac{2\left(1-r^{2}\right)}{1+\sqrt{1-r^{2}}}\right) & k=0, \ell=1, \\ c_{0, \ell-1}^{\lambda}(r)-\frac{r}{\ell-1}\left(c_{1, \ell}(r)-\frac{d}{d r} c_{0, \ell-1}^{\lambda}(r)\right) & k=0, \ell \geq 2, \\ r\left(c_{k-1,1}(r)-c_{k+1,1}(r)\right)(2 k)^{-1} & k \geq 1, \ell=0, \\ \left(c_{0, \ell-1}^{\lambda}(r)-c_{0, \ell}^{\lambda}(r)\right) r^{-1} & k=1, \ell \geq 1, \\ 2\left(c_{k-1, \ell-1}^{\lambda}(r)-c_{k-1, \ell}^{\lambda}(r)\right) r^{-1}-c_{k-2, \ell}^{\lambda}(r) & k \geq 2, \ell \geq 1,\end{cases}
$$

when $r \neq 0$. Also $c_{k, \ell}^{\lambda}(0)=0$.

Proof. In the case of $k=0, \ell=0$. Integrating, with respect to the variable $r$, the expression of $c_{1,1}(r)$, given in Corollary 22, and using the fact that $c_{1,1}(0)=0$ we obtain the expression for $c_{0,0}^{\lambda}$.

When $k=0, \ell=1$, we define the auxiliary function

$$
F(r, s)=\frac{-1}{2 \pi} \int_{-\pi}^{\pi} \frac{\log (1+r \cos \psi)}{1+s \cos \psi} d \psi
$$

for $r, s \in(-1,1)$. The derivative with respect to $r$ of $F(r, s)$, when we use Corollary 22 , gives

$$
\begin{aligned}
\frac{\partial F(r, s)}{\partial r} & =\frac{1}{2 \pi} \int_{-\pi}^{\pi} \frac{\cos \psi}{(1+r \cos \psi)(1+s \cos \psi)} d \psi \\
& =\frac{1}{s-r}\left(\frac{1}{2 \pi} \int_{-\pi}^{\pi} \frac{1}{1+r \cos \psi} d \psi-\frac{1}{2 \pi} \int_{-\pi}^{\pi} \frac{1}{1+s \cos \psi} d \psi\right) \\
& =\frac{1}{s-r}\left(c_{0,1}(r)-c_{0,1}(s)\right)=\frac{1}{s-r}\left(\frac{1}{\sqrt{1-r^{2}}}-\frac{1}{\sqrt{1-s^{2}}}\right)
\end{aligned}
$$

when $r \neq s$. Integrating the above relation with respect to $r$, taking into account that $F(0, s) \equiv 0$ and simplifying, we arrive at

$$
F(r, s)=\frac{1}{\sqrt{1-s^{2}}} \log \left(\frac{1-r s+\sqrt{1-r^{2}} \sqrt{1-s^{2}}}{1+\sqrt{1-s^{2}}}\right) .
$$

The expression of the statement comes from the limit $\lim _{s \rightarrow r} F(r, s)=c_{0,1}^{\lambda}(r)$.

When $k=0, \ell \geq 2$, we can write

$$
\begin{aligned}
\frac{d}{d r} c_{0, \ell-1}^{\lambda}(r) & =\frac{1}{2 \pi} \int_{-\pi}^{\pi} \frac{\cos \psi}{(1+r \cos \psi)^{\ell}} d \psi-\frac{\ell-1}{2 \pi} \int_{-\pi}^{\pi} \frac{\cos \psi \log (1+r \cos \psi)}{(1+r \cos \psi)^{\ell}} d \psi \\
& =c_{1, \ell}(r)-\frac{\ell-1}{2 \pi r} \int_{-\pi}^{\pi} \frac{(-1+1+r \cos \psi) \log (1+r \cos \psi)}{(1+r \cos \psi)^{\ell}} d \psi \\
& =c_{1, \ell}(r)-\frac{\ell-1}{r}\left(-c_{0, \ell}^{\lambda}(r)+c_{0, \ell-1}^{\lambda}(r)\right) .
\end{aligned}
$$

The expression of $c_{0, \ell}^{\lambda}$ follows if we solve the previous relation with respect to it. The case $k \geq 1, \ell=0$, follows if we integrate by parts. When $k=1, \ell \geq 1$, or $k \geq 2, \ell \geq 1$, we apply analogous arguments as in the proof of Proposition 21 . 
Proposition 24. The function $s_{k, \ell}^{\phi}$, defined in (40), is expressed as

$$
s_{k, \ell}^{\phi}(r)=\left\{\begin{array}{cc}
0 & k=0, \ell \geq 0, \\
\left(-c_{k, 1}(r)+\left(1-r^{2}\right)^{-\frac{1}{2}} c_{k, 0}(r)\right) k^{-1} & k \geq 1, \ell=0, \\
\left.-c_{0,1}^{\lambda}(r)+\left(1-r^{2}\right)^{-\frac{1}{2}} c_{0,0}^{\lambda}(r)\right) r^{-1} & k=1, \ell=1, \\
\frac{r}{\ell-1}\left(\frac{c_{2, \ell}(r)-c_{0, \ell}(r)}{2\left(1-r^{2}\right)}+s_{1, \ell-1}^{\phi}(r)\left(\frac{\ell-1}{r}-\frac{r}{1-r^{2}}\right)\right. & k=1, \ell \geq 2, \\
\left.+\frac{d}{d r} s_{1, \ell-1}^{\phi}(r)\right) & \\
2\left(s_{k-1, \ell-1}^{\phi}(r)-s_{k-1, \ell}^{\phi}(r)\right) r^{-1}-s_{k-2, \ell}^{\phi}(r) & k \geq 2, \ell \geq 1,
\end{array}\right.
$$

when $r \neq 0$. Also $s_{k, \ell}^{\phi}(0)=0$.

Proof. The cases $k \geq 1, \ell=0$ and $k=1, \ell=1$ follow by integration by parts. When $k=1, \ell \geq 2$ we can write

$$
\begin{aligned}
\frac{d}{d r} s_{1, \ell-1}^{\phi}(r)= & \frac{1}{2 \pi} \int_{-\pi}^{\pi} \frac{\sin \psi \frac{\partial}{\partial r} \phi(r, \psi)}{(1+r \cos \psi)^{\ell-1}} d \psi-\frac{\ell-1}{2 \pi} \int_{-\pi}^{\pi} \frac{\sin \psi \cos \psi \phi(r, \psi)}{(1+r \cos \psi)^{\ell}} d \psi \\
= & \frac{1}{2 \pi\left(1-r^{2}\right)} \int_{-\pi}^{\pi} \frac{\sin \psi\left(r \phi(r, \psi)+\sin \psi(1+r \cos \psi)^{-1}\right)}{(1+r \cos \psi)^{\ell-1}} d \psi \\
& -\frac{\ell-1}{2 \pi r} \int_{-\pi}^{\pi} \frac{\sin \psi(r \cos \psi+1-1) \phi(r, \psi)}{(1+r \cos \psi)^{\ell}} d \psi \\
= & \frac{r}{1-r^{2}} s_{1, \ell-1}^{\phi}(r)+\frac{1}{1-r^{2}} \frac{c_{0, \ell}(r)-c_{2, \ell}(r)}{2} \\
& -\frac{\ell-1}{r} s_{1, \ell-1}^{\phi}(r)+\frac{\ell-1}{r} s_{1, \ell}^{\phi}(r) .
\end{aligned}
$$

Here we have used the identity $2 \sin ^{2} \psi=1-\cos (2 \psi)$. The relation of the statement is followed by isolating $s_{1, \ell}^{\phi}(r)$ in the above equality. The proof ends, when $k \geq$ $2, \ell \geq 1$, if we use an analogous argument in the corresponding case in the proof of Proposition 21.

\section{ACKNOWLEDGEMENTS}

The first author is supported by the MINECO/FEDER MTM2011-22751 grant and the second author is supported by the MINECO/FEDER MTM2008-03437, UNAB10-4E-378 and the Generalitat de Catalunya 2009SGR410 grants.

We would like to thank Armengol Gasull for his comments and suggestions.

\section{REFERENCES}

[1] M. Álvarez, A. Gasull, and J. Yu. Lower bounds for the number of limit cycles of trigonometric Abel equations. J. Math. Anal. Appl., 342(1):682-693, 2008.

[2] A. Buică, A. Gasull, and J. Yang. The third order Melnikov function of a quadratic center under quadratic perturbations. J. Math. Anal. Appl., 331(1):443-454, 2007.

[3] A. Buică, J. Giné, and J. Llibre. A second order analysis of the periodic solutions for nonlinear periodic differential systems with a small parameter. Phys. D, 241(5):528-533, 2012.

[4] J. Chavarriga and M. Sabatini. A survey of isochronous centers. Qual. Theory Dyn. Syst., 1(1):1-70, 1999. 
[5] G. Chen, C. Li, J. Llibre, and C. Liu. The cyclicity of period annuli of some classes of reversible quadratic systems. Discrete Contin. Dyn. Syst., 16(1):157-177, 2006.

[6] C. Chicone and M. Jacobs. Bifurcation of limit cycles from quadratic isochrones. J. Differential Equations, 91(2):268-326, 1991.

[7] C. Christopher and C. Li. Limit cycles of differential equations. Advanced Courses in Mathematics. CRM Barcelona. Birkhäuser Verlag, Basel, 2007.

[8] B. Coll, C. Li, and R. Prohens. Quadratic perturbations of a class of quadratic reversible systems with two centers. Discrete Contin. Dyn. Syst., 24(3):699-729, 2009.

[9] J. P. Françoise. Successive derivatives of a first return map, application to the study of quadratic vector fields. Ergodic Theory Dyn. Syst., 16(1):87-96, 1996.

[10] J. P. Françoise. Parametric centers for trigonometric Abel equations. J. Dyn. Differ. Equations, 20(4):777-786, 2008.

[11] A. Garijo, A. Gasull, and X. Jarque. Simultaneous bifurcation of limit cycles from two nests of periodic orbits. J. Math. Anal. Appl., 341:813-824, 2008.

[12] A. Gasull, J. Lázaro, and J. Torregrosa. On the Chebyshev property for a new family of functions. J. Math. Anal. Appl., 387:631-644, 2012.

[13] A. Gasull, C. Li, and J. Torregrosa. A new Chebyshev family with applications to Abel equations. J. Differential Equations, 252(2):1635-1641, 2012.

[14] L. Gavrilov. Higher order Poincaré-Pontryagin functions and iterated path integrals. Ann. Fac. Sci. Toulouse Math. (6), 14(4):663-682, 2005.

[15] L. Gavrilov and I. D. Iliev. Second-order analysis in polynomially perturbed reversible quadratic Hamiltonian systems. Ergodic Theory Dynam. Systems, 20(6):1671-1686, 2000.

[16] L. Gavrilov and I. D. Iliev. The displacement map associated to polynomial unfoldings of planar Hamiltonian vector fields. Amer. J. Math., 127(6):1153-1190, 2005.

[17] J. Giné, M. Grau, and J. Llibre. Averaging theory at any order for computing periodic orbits. Phys. D, 250:58-65, 2013.

[18] I. D. Iliev. Higher-order Melnikov functions for degenerate cubic Hamiltonians. Adv. Differential Equations, 1(4):689-708, 1996.

[19] I. D. Iliev. On second order bifurcations of limit cycles. J. London Math. Soc. (2), 58(2):353$366,1998$.

[20] A. Jebrane, P. Mardešić, and M. Pelletier. A note on a generalization of Françoise's algorithm for calculating higher order Melnikov functions. Bull. Sci. Math., 128(9):749-760, 2004.

[21] A. Jebrane and H. Żołądek. A note on higher order Melnikov functions. Qual. Theory Dyn. Syst., 6(2):273-287, 2005.

[22] S. Karlin and W. Studden. Tchebycheff systems: With applications in analysis and statistics. Pure and Applied Mathematics. Vol. 15. New York-London-Sydney: Intersecience Publishers a division of John Wiley \& Sons. XVIII, 586 pp., 1966.

[23] C. Li, W. Li, J. Llibre, and Z. Zhang. Linear estimate for the number of zeros of Abelian integrals for quadratic isochronous centres. Nonlinearity, 13(5):1775-1800, 2000.

[24] C. Li, W. Li, J. Llibre, and Z. Zhang. Linear estimate of the number of zeros of Abelian integrals for a class of integrable non-Hamiltonian systems. Nonlinear Anal., 47(7):45274531, 2001.

[25] C. Liu. The cyclicity of period annuli of a class of quadratic reversible systems with two centers. J. Differential Equations, 252(10):5260-5273, 2012.

[26] J. Llibre, J. S. Pérez, and J. A. Rodríguez. Averaging analysis of a perturbated quadratic center. Nonlinear Anal. Ser. A, 46(1):45-51, 2001.

[27] J. Llibre and G. Świrszcz. On the limit cycles of polynomial vector fields. Dyn. Contin. Discrete Impuls. Syst. Ser. A Math. Anal., 18(2):203-214, 2011.

[28] J. Llibre and J. Yu. On the upper bound of the number of limit cycles obtained by the second order averaging method. Dyn. Contin. Discrete Impuls. Syst. Ser. B Appl. Algorithms, 14(6):841-873, 2007.

[29] W. S. Loud. Behavior of the period of solutions of certain plane autonomous systems near centers. Contributions to Differential Equations, 3:21-36, 1964.

[30] P. Mardešić, C. Rousseau, and B. Toni. Linearization of isochronous centers. J. Differ. Equations, 121(1):67-108, 1995. 
[31] L. S. Pontryagin. Über Autoschwingungssysteme, die den Hamiltonschen nahe liegen. Physikal. Z. Sowjetunion, 6:25-28, 1935.

[32] R. Prohens and J. Torregrosa. Shape and period of limit cycles bifurcating from a class of Hamiltonian period annulus. Nonlinear Anal. Ser. A, 81:130-148, 2013.

[33] J. Torregrosa. Singulars points and periodic orbits for a vector fields. PhD thesis, Universitat Autònoma de Barcelona, 1998. (In Catalan).

Dept. de Matemàtiques i Informàtica, Escola Politècnica Superior, Universitat de les Illes Balears. 07122, Palma de Mallorca. Spain

E-mail address: rafel.prohens@uib.cat

Dept. de Matemàtiques, Universitat Autònoma de Barcelona, Edifici C. 08193 Bellaterra, Barcelona. Spain

E-mail address: torre@mat.uab.cat 